Review article

\title{
Factors affecting polyhydroxyalkanoates biodegradation in soil
}

\author{
Miguel Fernandes*, Andreia Salvador, Madalena M. Alves, António A. Vicente \\ Centre of Biological Engineering, University of Minho, Campus de Gualtar, Braga 4710-057, Portugal
}

\section{A R T I C L E I N F O}

\section{Article history:}

Received 12 July 2020

Revised 16 October 2020

Accepted 19 October 2020

Available online 19 October 2020

\section{Keywords:}

Soil biodegradation

Polyhydroxyalkanoates

Biodegradable plastics

PHA degraders

\begin{abstract}
A B S T R A C T
Polyhydroxyalkanoates (PHAs) are polymers with widespread applications, from medical devices to packaging. PHAs can be biodegradable in natural environments, such as soil, but the blend of PHA with other materials can change the polymer properties and consequently affect the biodegradation process. The composition of the microbial communities in soil also significantly affects the biodegradation, but other factors such as temperature, $\mathrm{pH}$, and soil moisture, can also be determinant. These ecological and physic/chemical factors change in different seasons and in different soil layers. It is essential to know how these factors influence the PHAs' biodegradation to understand the impact of PHAs in nature. This review compiles the results on PHA polymers and PHA blends biodegradation, with focus on laboratory tests. The main factors affecting PHA's biodegradation in soil, both in laboratory tests and in the environment are also discussed.
\end{abstract}

(C) 2020 Elsevier Ltd. All rights reserved.

\section{Introduction}

The so-called "conventional" plastics are synthetic and semisynthetic polymeric compounds that are produced mainly from fossil carbon sources such as crude oil and natural gas. Specific characteristics of plastics, including durability, processability, and low production price, have led to their widespread use in extensive and varied applications worldwide since the $20^{\text {th }}$ century. Packages are the major market sector in which plastics are used, and are mostly conceived for immediate disposal [1]. This fact represents an environmental problem, as conventional plastics are very resistant to biological degradation. Packages usually consist of conventional plastics, such as polyethylene (PE) or polypropylene (PP), which are recalcitrant and thus accumulate in landfills and marine environments [2]. They cause the contamination of drinking water and the ingestion of disintegrated waste plastics by marine wildlife, introduce them in the food chain, which has inestimable consequences in human health, threaten global biodiversity and impact the environment [3]. These adverse effects and the excessive use of plastics led to significant challenges for waste treatment processes. Extensive efforts have been developed to create alternative plastic materials that can be competitive in economic terms, made of renewable feedstocks, and that can, preferentially, undergo biodegradation, without causing harmful effects in the envi-

\footnotetext{
* Corresponding author.

E-mail addresses: miguel.fernandes@ceb.uminho.pt (M. Fernandes), asalvador@ceb.uminho.pt (A. Salvador), madalena.alves@deb.uminho.pt (M.M. Alves), avicente@deb.uminho.pt (A.A. Vicente).
}

ronment [4]. Nowadays, existing plastics can be classified into four groups, as indicated in Fig. 1, regarding whether they are considered biodegradable and the source of the feedstock used for their production [5].

Conventional plastics, also known as petro-based or synthetic plastic, are generally derived from non-renewable resources and are non-biodegradable [5]. These fossil-based plastics may be biodegradable, and in that case, they are called biodegradable plastics. The group of bio-based plastics refers to those plastics synthesized from natural resources or biomass. These plastics can either be biodegradable or not, and are designated as biodegradable bio-based plastics (e.g., Polylactic acid (PLA), polyhydroxyalkanoates (PHAs)) or non-biodegradable bio-based plastics (e.g., bio$\mathrm{PE})$, respectively [5].

The PHA family of polymers is considered biodegradable, nontoxic, environmentally friendly, and can be produced from renewable resources [6,7]. Nowadays, PHA polymers have the potential to compete with conventional plastics due to their characteristics that can include a high degree of polymerization crystallinity and insolubility in water. [6,7]. PHAs are biopolyesters that naturally accumulate intracellularly in a wide range of microorganisms, such as bacterial and archaeal cells. They are produced through the fermentation of sugars and lipids and function mainly as energy and carbon storage compounds [8]. These polyesters are produced when bacterial growth is limited by depletion of nitrogen or phosphorous, or when an excess of carbon source is available [9]. For example, Sphingopyxis chilensis survived during carbon starvation or frozen conditions by consuming the accumulated PHAs [10]. PHAs are composed of 3-hydroxy fatty acid monomers, which 


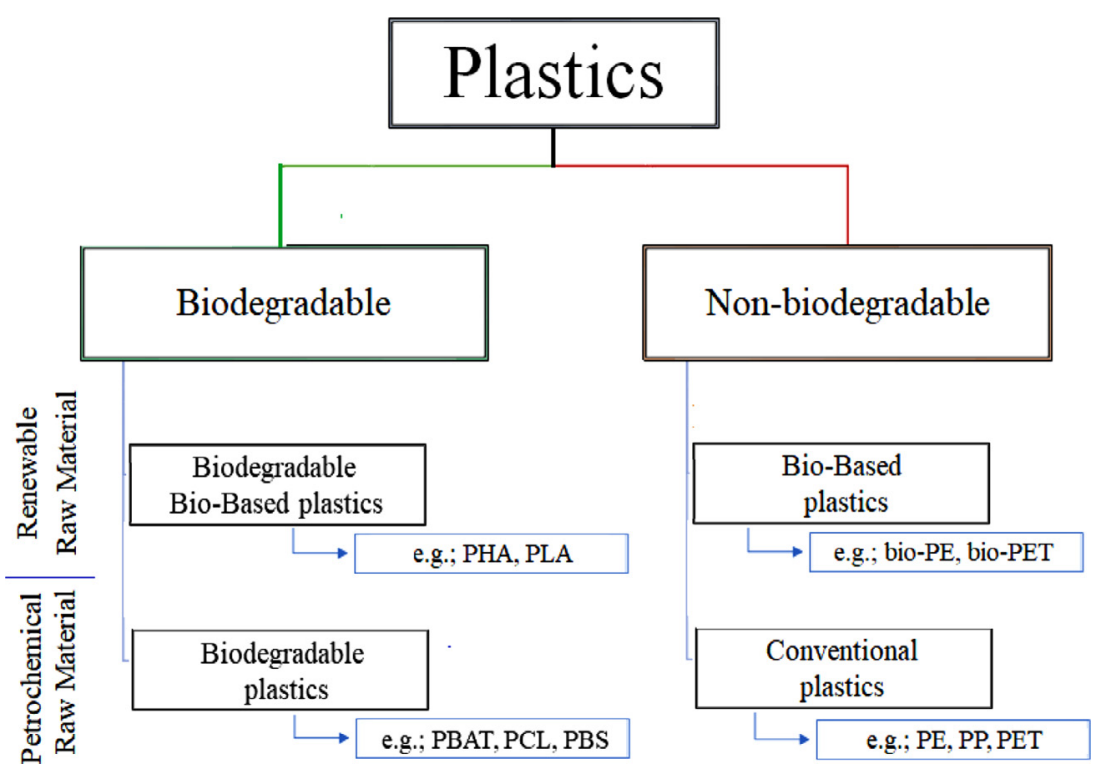

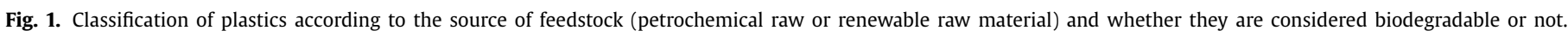

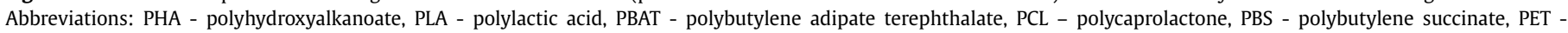
polyethylene terephthalate, PE - polyethylene, PP - polypropylene, bio-PE - bio based Polyethylene, bio-PET - bio based Polyethylene terephthalate.

form linear, head-to-tail polyester molecules. They are typically polymers of $10^{3}$ to $10^{4}$ monomers, which accumulate as inclusions of $0.2-0.5 \mu \mathrm{m}$ in diameter [11]. These inclusions or granules are synthesized and stored by microorganisms and cause no harmful effects to the hosts. Depending on the number of carbons in their monomeric constituents (3-hydroxyalkanoate units), PHAs can be classified as short-chain-length (scl-PHA), containing monomers of 3-5 carbon atoms, and medium-chain-length (mclPHA), with monomers containing more than 6 carbon atoms [12]. The scl-PHAs are considered thermoplastic due to their relatively high crystallinity and their properties that resemble those of some petrochemical-based polymers, whereas mcl-PHAs present minimal crystallinity and exhibit elastomeric and/or free-flowing properties [12].

The existence of PHAs in bacteria has been known since 1926 when Lemoigne reported the formation of poly(3polyhydroxybutyrate) (PHB) in the cytoplasm of the bacteria Bacillus megaterium [13]. The PHA bioaccumulation is common in the domains Bacteria and Archaea with PHA producing organisms belonging to more than 70 genera $[14,15]$. Most species of bacteria that produce PHA are Gram-negative from genera Azohydromonas, Burkholderia, Pseudomonas, and Cupriavidus [14] and Cupriavidus necator (formerly Wautersia eutropha) is the most widely studied [16]. PHA production in Gram-positive bacteria has been described in genera Bacillus, Caryophanon, Clostridium, Corynebacterium, Micrococcus, Microlunatus, Microcystis, Nocardia, Rhodococcus, Staphylococcus, and Streptomyces [14]. PHA is also found in Archaea but is limited to Haloarchaea and include the genera Haloferax, Halalkalicoccus, Haloarcula, Halobacterium, Halobiforma, Halococcus, Halopiger, Haloquadratum, Halorhabdus, Halorubrum, Halostagnicola, Haloterrigena, Natrialba, Natrinema, Natronobacterium, Natronococcus, Natronomonas, and Natronorubrum [17].

Around 150 different PHA monomers have been identified so far [18]. The tendency is that this number will rapidly increase due to the synthesis of the novel PHAs as a result of chemical or physical modifications of naturally-occurring PHA [19]. PHAs are produced biologically from several carbon sources, including gases such as methane, n-alcohols such as ethanol, n-alkanes such as octanes, n-alkanoic acids such as oleic acid, and saccharides such as fructose or glucose [20-22]. Waste streams as for example, plant oil mills effluents, frying oil waste, vinegar waste, waste fats, food waste, and agricultural waste, have also been reported as alternative carbon sources for PHAs biosynthesis [23]. Indeed, exhaustive research has been conducted to expand PHA production from lower-cost carbon sources and waste in order to reduce production costs [24]. In this context, the co-culturing of different microbial strains has been applied as a strategy to reduce production costs. In these cases, the first microorganism transforms the carbon substrate into a metabolite that can be later consumed by the second microorganism for PHA production. For example, Cupriavidus necator cannot efficiently metabolize sugars, whey or starchy waste, but when cultivated together with lactic acid-producing bacteria, those substrates can be transformed into lactate, that can be then used by $C$. necator to produce PHAs [25].

The intracellular PHA granules produced by the bacteria need to be extracted, and for that purpose, the bacterial cells are usually separated from the medium by centrifugation. Organic solvents such as acetone, chloroform, methylene chloride, or dichloroethane are utilized to lyse the cells and recover the intracellular PHA [26,27]. Digestion methods with sodium hypochlorite [28] or enzymatic digestion procedures using, for example, EDTA or SDS [29,30] are alternatives to organic solvents.

Polyhydroxybutyrate (PHB) is the most common and wellstudied polymer within the PHA family [31]. Other polymers belonging to the PHA class include poly(4-hydroxybutyrate) (P4HB), poly(3-hydroxyvalerate) (PHV), poly(3-hydroxyhexanoate) (PHH), poly(3-hydroxyoctanoate) (PHO), and their copolymers.

Several microorganisms are able to decompose PHAs, by using intracellular depolymerase enzymes and use it as an energy and carbon source [32,33]. In the environment, various microorganisms produce and release extracellular enzymes that can degrade PHAs [34], such as PHA hydrolases and PHA depolymerases [35]. Mergaert and Swings [36] identified 695 microbial species capable of degrading PHB. Usually, depolymerases catalyze the hydrolysis originating free $\mathrm{D}(-)-3$-hydroxybutyrate that is then oxidized to acetoacetate by a NAD-specific dehydrogenase. NADH, pyruvate, and 2-oxoglutarate are known to inhibit this enzyme. The acetoacetate is finally converted to acetoacetyl-CoA by an acetoacetate/succinate CoA transferase. Thus, acetoacetyl-CoA is simulta- 
neously a precursor of PHB synthesis and a product of PHB degradation [37].

PHA polymers may present different monomers composition, physicochemical properties, size, and structure because they can be produced by different microorganisms and from diverse substrates [38]. The lower environmental impact of PHAs turns them an ideal alternative for petrochemical polymers, particularly in packaging and coating applications [39]. Nowadays, PHAs are mainly applied in packaging, in the form of containers and films [40] but also in the medical field. For example, PHB can be used as repair patches, orthopaedic pins, adhesion barriers, stents, nerve guides, and bone marrow scaffolds, since it is compatible with mammal's blood and tissues [41].

\section{PHAs biodegradation in soil}

The biodegradation of PHA has been studied in the last 3 decades in several types of soils and under different conditions. According to the definition of the International Standardization Organization (ISO), plastics need to undergo significant changes in their chemical structure by the activity of naturally occurring microorganisms to be considered biodegradable [42]. On the other hand, the European Standardization Committee (CEN) is stricter and considers that a biodegradable plastic needs to be converted into microbial metabolic products. Concerning aerobic biodegradation of plastics in soil, the most used standard testing methods for laboratories are the ASTM D5988-18 [43], ISO 17556 [44], and the French and Italian norms NF U 52-001 [45] and UNI 11462 [46], respectively [47]. However, the NF U52-001 [45] was recently superseded by EN 17033 [48] for "Plastics - Biodegradable mulch films for use in agriculture and horticulture e Requirements and test methods". EN 17033 [48] was created using the methodology of ISO 17556. The ISO 17566 uses a method where the $\mathrm{CO}_{2}$ production is measured in a system aerated with continuous $\mathrm{CO}_{2}$-free air [49]. This method is equivalent to the ASTM D5988 for determining the aerobic biodegradation of plastic materials in the soil. In this method, closed flasks (bioreactors) are used and are aerated only periodically. The evolved $\mathrm{CO}_{2}$ from the sample is trapped in an alkaline solution (BaOH or $\mathrm{KOH})$ and measured by titration [49]. Alternatively, the biochemical oxygen demand can also be implemented [44].

A significant amount of plastic waste can be found on soil. The soil pollution problem, caused in a greater extent by plastic waste, can be somewhat alleviated by the manufacture and use of advanced bio-based plastics that can be biodegradable [47]. The high diversity of microorganisms present in soil increases the possibility of finding microbes capable of degrading biodegradable plastic waste. Because soil may hold microorganisms with this ability, it has been considered an environment with an excellent capacity for degrading PHA [50]. Two different studies estimated that the percentage of bacteria capable of degrading scl-PHA is between 0.8 to $11.0 \%$, and 2 to $18 \%$ of the total colonies formed from soil plating [51,52]. Table 1 summarizes the laboratory biodegradation tests conducted with PHA polymers. Gómez and Michel [5] tested the biodegradability of several commercially available alternative materials for conventional plastic, according to the ASTM D598803. During incubation in soil for 660 days (Table 1 ), $\mathrm{CO}_{2}$ production was monitored, and the results showed that the maximum biodegradability was obtained for PHA films (around $70 \%$ ), which was not statistically different from that of cellulose paper (control). SEM analysis presented considerable disintegration of PHA-based plastic compared to the other materials tested [5]. Scanning electron microscopy (SEM) is often considered a valuable tool to study colonization and biodegradation of PHA films. For example, it was utilized to evaluate the biodegradability of PHBV by soil microorganisms, since the polymer deterioration generally occurs through surface erosion, due to microbial activity [53-55]. In natural soils, polymers from the PHA family present higher biodegradation rates (evaluated by weight loss) when compared to other polymers such as poly-DL-lactide and ethyl cellulose [56]. These results may be due to higher biofilm development on PHA plastic films [56].

PHB tested in different laboratories using the ASTM D5988 and some specifications of the ISO 17556 for plastics exhibit a similar degree of biodegradation in both natural and standard soils (Table 1). Other polymers that resist more to biodegradation, such as PBSeT and PBSe, present some differences, being biodegraded slowly in natural soils [49]. In another work, were the ASTM 5988 system was used, PHA biodegradation results were significantly different between 2 laboratories [57]. These authors also discovered that PHA degraded faster at $25{ }^{\circ} \mathrm{C}$ than at $37{ }^{\circ} \mathrm{C}$ in both laboratories. However, this may be due to higher biomass build-up and consequent carbon retention [57]. Kim et al. [58] demonstrated that the biodegradation of PHB was higher in different soils at 37 ${ }^{\circ} \mathrm{C}$ than at $28{ }^{\circ} \mathrm{C}$, and the worst temperature for biodegradation was the highest tested, $60{ }^{\circ} \mathrm{C}$ (Table 1) [58]. PHBV (copolymer of 3-hydroxybutyrate and 3-hydroxyvalerate) degraded at a faster rate at $30^{\circ} \mathrm{C}$ than at $52^{\circ} \mathrm{C}$ in soil under aerobic conditions [59]. Mergaert et al. [60] discovered that the biodegradation rate in soils (laboratory testing) of PHB and PHBV (10 mol \% HV) was enhanced at higher temperatures $\left(40^{\circ} \mathrm{C}\right)$, and similar results were obtained by other authors [61]. The differences among these studies may be related to different microbial activities in soil, which are strongly influenced by temperature.

Several studies have demonstrated that other conditions and properties of the PHA materials can also affect the biodegradation rate. Specifically, copolymers (polymers derived from more than one species of monomer) are degraded at a faster rate than the homopolymers, although the differences varied widely between soils with several $\mathrm{pH}$ values (neutral or acid) and temperatures. Copolymers (e.g., PHBV) usually have a higher degradation rate compared to homopolymers (e.g., PHB) of the PHA family [60]. This higher biodegradation capability is attributed to the surface morphology of copolymers, which combines a low crystallinity and a porous surface, allowing a faster degradation [62].

The biodegradation of PHAs with different chemical compositions was tested in soil for 35 days. These polymers could be ordered as following according to the biodegradation rate (from higher biodegradation rate to lower biodegradation rate): $\mathrm{PHB} / 4 \mathrm{HB}$ $>$ PHB3HHx $>$ PHBV $>$ PHB. In this work, biodegradation was higher for all polymers at $28{ }^{\circ} \mathrm{C}$ than at $21{ }^{\circ} \mathrm{C}$ (Table 1) [31]. PHB films, the most crystalline ones, remained nearly unchanged, suggesting that all regions (crystalline and amorphous) were degraded at similar rates [30]. But for the 3 copolymers, the crystallinity increased, demonstrating that the amorphous regions were degraded at higher rates. Other works, including studies performed in natural environments (where the biodegradation was evaluated by weight loss), present the same pattern, indicating that PHB is more resistant to biodegradation due to its high crystallinity in comparison with the copolymers $[50,63,64]$.

In these works, plastics biodegradation has been evaluated not only by $\mathrm{CO}_{2}$ production or $\mathrm{O}_{2}$ consumption but also by weight loss or loss of mechanical properties over time. Although some works use weight loss to assess biodegradation in soil, sometimes it is difficult to adequately clean the samples following soil burial and obtain the exact weight loss [65]. Sometimes it is impossible to use weight loss in later stages of the tests due to a high biodegradation level of the material, hindering a proper weight evaluation [66]. The tested material may be biodegradable in testing conditions at a specific rate, however because the properties in real environments vary extensively (e.g., microbial communities' composition and environmental conditions), the results may be not representative of those environments. 
Table 1

Laboratory soil biodegradation tests for PHA polymers.

\begin{tabular}{|c|c|c|c|c|c|c|c|}
\hline Type of Plastic & $\begin{array}{l}\text { Type of } \\
\text { material }\end{array}$ & $\begin{array}{l}\text { Type of } \\
\text { environment }\end{array}$ & Conditions & Test method & $\begin{array}{l}\text { Biodegradation } \\
(\%)\end{array}$ & $\begin{array}{l}\text { Length of test } \\
\text { (days) }\end{array}$ & Reference \\
\hline $\begin{array}{l}\text { PHB } \\
\text { PHB/CAB (50 } \\
\% / 50 \%)\end{array}$ & Films & Garden soil & Not indicated & Weight loss & $\begin{array}{l}64.3 \\
31.5\end{array}$ & 180 & [1] \\
\hline PHA & Films & $\begin{array}{l}\text { Alluvial-type } \\
\text { soil }\end{array}$ & $35 \%$ soil moisture & Weight loss & $\sim 35$ & 60 & [67] \\
\hline $\begin{array}{l}\text { PHA/Rice Husk } \\
(60 / 40 \mathrm{wt} \%)\end{array}$ & & & & & $>90$ & & \\
\hline $\begin{array}{l}\text { PHA-g-AA/Rice } \\
\text { Husk }(60 / 40 \\
\text { wt } \%)\end{array}$ & & & & & 84 & & \\
\hline $\begin{array}{l}\text { PHBV (HV of } \\
12 ; 43 ; 47 ; 52 ; \\
64 ; 72 \mathrm{~mol} \%)\end{array}$ & Powder & $\begin{array}{l}\text { Soil/compost } \\
(90 \% / 10 \%)\end{array}$ & $\begin{array}{l}25{ }^{\circ} \mathrm{C}, 65 \% \text { humidity, } \\
80 \% \text { soil moisture } \\
\text { holding capacity }\end{array}$ & $\mathrm{CO}_{2}$, ASTM D5988-03 & $\begin{array}{l}67 ; 54 ; 48 ; 62 ; \\
49 ; 49 ;\end{array}$ & 112 & {$[68]$} \\
\hline PHA & Films & $\begin{array}{l}43 \% \text { certified } \\
\text { organic topsoil, } \\
43 \% \text { no-till } \\
\text { farm soil, and } \\
14 \% \text { sand }\end{array}$ & $20^{\circ} \mathrm{C}, 60 \%$ moisture & $\mathrm{CO}_{2}$, ASTM D5988-03 & 74.2 & 660 & [5] \\
\hline $\begin{array}{l}\text { PHBV/WF } \\
(50 / 50 \text { wt \%) }\end{array}$ & Films & $\begin{array}{l}\text { Soil } \\
\text { sub-tropical }\end{array}$ & $\begin{array}{l}80 \% \text { of soil water } \\
\text { holding capacity }\end{array}$ & $\mathrm{CO}_{2}$ ASTM D5988-03 & 36 & 330 & [69] \\
\hline $\begin{array}{l}\text { PHBV/WF } \\
(80 / 20 \text { wt \%) }\end{array}$ & & & & & 35 & & \\
\hline PHBV & Films & Garden soil & $\begin{array}{l}23^{\circ} \mathrm{C}, 21 \% \text { soil } \\
\text { humidity }\end{array}$ & Weight loss & $\sim 20$ & 180 & {$[66]$} \\
\hline $\begin{array}{l}\text { PHBV /Sep } \\
\text { straw }\end{array}$ & & & & & $\sim 23$ & & \\
\hline PHB & Films & Garden soil & Not described & Weight loss & 100 & 180 & {$[32]$} \\
\hline PHB + Acrylate & & & & & 10 & & \\
\hline PHBV & Films & Garden Soil & Room temperature & Weight loss & $\begin{array}{l}3 \\
18\end{array}$ & $\begin{array}{l}30 \\
30\end{array}$ & {$[70]$} \\
\hline \multicolumn{8}{|l|}{$\begin{array}{l}\text { PDLLA/PHBV/PEG } \\
(30 / 70 / 20 \mathrm{wt} \\
\%)\end{array}$} \\
\hline PHB & Films & $\begin{array}{l}\text { Hardwood, } \\
\text { Pinewood, } \\
\text { Sandy, Clay, } \\
\text { Loamy soil }\end{array}$ & $\begin{array}{l}28^{\circ} \mathrm{C}, \mathrm{pH} 3.9 ; 3.5 ; 6.5 ; \\
7.1 ; 3.3,14-22 \% \text { water } \\
\text { content }\end{array}$ & Weight loss & $\begin{array}{l}77 ; 74 ; 88 ; 78 ; \\
93\end{array}$ & 200 & [61] \\
\hline $\begin{array}{l}\text { PHBV (HV of } \\
10 \%)\end{array}$ & & & & & $\begin{array}{l}67 ; 64 ; 90 ; 53 ; \\
69\end{array}$ & & \\
\hline PHB & Films & Garden soil & $\mathrm{pH} 7.3 \pm 0.2$ & Weight loss & 83 & 77 & [71] \\
\hline PHBV & Films & $\begin{array}{l}\text { Organic } \\
\text { compound } \\
\text { humidified } \\
\text { using poultry } \\
\text { feces and } \\
\text { plant-origin } \\
\text { organic } \\
\text { materials }\end{array}$ & $\begin{array}{l}\mathrm{pH} 6,40 \% \text { maximum } \\
\text { humidity, nitrogen of } 1 \\
\%, \text { minimum organic } \\
\text { material of } 40 \%, \mathrm{C}: \mathrm{N} \\
\text { maximum of } 18: 1\end{array}$ & Weight loss & $9.77 \pm 2.77$ & 90 & {$[72]$} \\
\hline $\begin{array}{l}\text { PHBV/WF } \\
(80 / 20 \text { wt \%) }\end{array}$ & & & & & $25.55 \pm 4.05$ & 60 & \\
\hline $\begin{array}{l}\text { PHBV/Sisal } \\
\text { fiber }(80 / 20 \text { wt } \\
\%)\end{array}$ & & & & & $25.02 \pm 8.23$ & 60 & \\
\hline PHBV-F0 & $\begin{array}{l}\text { Particles } \\
\text { around 1-2mm }\end{array}$ & $\begin{array}{l}\text { Soil park ( } 2.3 \\
\text { wt \% of organic } \\
\text { matter, } 16.85 \\
\text { wt \% of clay, } \\
26.85 \text { wt } \% \text { of } \\
\text { lime, and } 56.3 \\
\text { wt \% of sand) }\end{array}$ & $\begin{array}{l}28{ }^{\circ} \mathrm{C}, \mathrm{pH} 6.8,80 \% \text { of } \\
\text { the soil water } \\
\text { retention capacity }\end{array}$ & $\mathrm{CO}_{2}$, ASTM D5988-96 & 100 & 75 & [73] \\
\hline PHBV-SF & & & & & 100 & 79 & \\
\hline PHBV-PF & & & & & 100 & 87 & \\
\hline $\begin{array}{l}\text { PHBV (HV of } \\
3 \% \text { ) }\end{array}$ & & & & & 91 & 123 & \\
\hline $\begin{array}{l}\text { PHB-starch } \\
(75 / 25 \text { wt \%) } \\
\text { PHB-starch } \\
(60 / 40 \text { wt \%) } \\
\text { PHB-starch } \\
(40 / 60 \text { wt \%) }\end{array}$ & Films & Garden soil & $\begin{array}{l}25 \pm 2^{\circ} \mathrm{C}, \mathrm{pH} 6.8 \text {, soil } \\
\text { water content } 45 \%\end{array}$ & Weight loss & $50-60$ & $14-21$ & [74] \\
\hline $\begin{array}{l}\text { PHA (peanut } \\
\text { oil) }\end{array}$ & Films & $\begin{array}{l}\text { Commercial } \\
\text { soil containing } \\
39 \% \text { average } \\
\text { organic matter }\end{array}$ & $\begin{array}{l}23^{\circ} \mathrm{C} \pm 4^{\circ} \mathrm{C}, \mathrm{pH} 6.8,33 \\
\% \text { soil moisture }\end{array}$ & Weight loss & 75 & 80 & [75] \\
\hline
\end{tabular}


Table 1 (continued)

\begin{tabular}{|c|c|c|c|c|c|c|c|}
\hline Type of Plastic & $\begin{array}{l}\text { Type of } \\
\text { material }\end{array}$ & $\begin{array}{l}\text { Type of } \\
\text { environment }\end{array}$ & Conditions & Test method & $\begin{array}{l}\text { Biodegradation } \\
(\%)\end{array}$ & $\begin{array}{l}\text { Length of test } \\
\text { (days) }\end{array}$ & Reference \\
\hline PHB & Films & $\begin{array}{l}\text { Soil of } \\
\text { temperate zone } \\
\text { of Siberia, with } \\
\text { high total } \\
\text { exchangeable } \\
\text { bases } \\
(40.0-45.2 \\
\text { mequiv } / 100 \mathrm{~g}) \text {, } \\
\text { and with } \\
\text { nitrate } \\
\text { nitrogen } \\
\mathrm{N}-\mathrm{NO}_{3} 6, \mathrm{P}_{2} \mathrm{O}_{5} \\
60 \text {, and } \mathrm{K}_{2} \mathrm{O} \\
220 \mathrm{mg} / \mathrm{kg} \text { soil }\end{array}$ & $\begin{array}{l}28^{\circ} \mathrm{C}, \mathrm{pH} 7.1-7.8 \text { and } \\
50 \% \text { soil moisture }\end{array}$ & Weight loss & 93 & 35 & [31] \\
\hline PHBV & & & & & 100 & & \\
\hline РНВ3HНx & & & & & 100 & & \\
\hline PHB/4HB & & & & & 100 & 28 & \\
\hline PHB & $\begin{array}{l}\text { Powder- } \\
\text { Granules }\end{array}$ & $\begin{array}{l}\text { Agro- } \\
\text { transformed } \\
\text { soil with } 280 \\
\mathrm{mg} / \mathrm{kg} \text { of } \\
\text { phosphorus } \\
\text { and } 250 \mathrm{mg} / \mathrm{kg} \\
\text { of potassium }\end{array}$ & $\begin{array}{l}25^{\circ} \mathrm{C} ; 50 \% \text { soil } \\
\text { moisture content }\end{array}$ & Weight loss & $32-31.6$ & 35 & [76] \\
\hline PHB/peat & & & & & $43,6-53.6$ & & \\
\hline PHB/clay & & & & & $36-26$ & & \\
\hline PHB/WF & & & & & $33-23$ & & \\
\hline \multirow[t]{3}{*}{ PHB } & Films & Forest soil & $28^{\circ} \mathrm{C} ; 37^{\circ} \mathrm{C} ; 60^{\circ} \mathrm{C}$ & Weight loss & $\begin{array}{l}10.5 \pm 1.4 ; 7.1 \\
\pm 0.7 ; 4.9 \pm \\
0.3\end{array}$ & 25 & [58] \\
\hline & & Sandy soil & $28^{\circ} \mathrm{C} ; 37^{\circ} \mathrm{C} ; 60^{\circ} \mathrm{C}$ & & $\begin{array}{l}5.8 \pm 0.4 ; 10.0 \\
\pm 1.2 ; 4.5 \pm \\
0.5\end{array}$ & & \\
\hline & & Farm soil & $28^{\circ} \mathrm{C} ; 37^{\circ} \mathrm{C} ; 60^{\circ} \mathrm{C}$ & & $\begin{array}{l}41.3 \pm 3.7 \\
68.8 \pm 4.8 \\
14.8 \pm 1.2\end{array}$ & & \\
\hline \multirow[t]{2}{*}{ PHA (MirelTM) } & Films & $\begin{array}{l}\text { Soil from the } \\
\text { experimental } \\
\text { field in Spata }\end{array}$ & $\begin{array}{l}30^{\circ} \mathrm{C}, 40 \% \text { water } \\
\text { content }\end{array}$ & $\begin{array}{l}\text { Biological oxygen } \\
\text { demand }\end{array}$ & 26.3 & 10 & [77] \\
\hline & & & $\begin{array}{l}40^{\circ} \mathrm{C}, 40 \% \text { water } \\
\text { content }\end{array}$ & & 49.5 & 12 & \\
\hline \multirow[t]{2}{*}{$\begin{array}{l}\text { PHBV (HV of } \\
10 \%)\end{array}$} & Films & $\begin{array}{l}\text { Soil from the } \\
\text { Nagoya } \\
\text { University } \\
\text { Farm and } 2 \% \\
(\mathrm{w} / \mathrm{w}) \text { of } \\
\text { Farmyard } \\
\text { manure }\end{array}$ & $\begin{array}{l}30^{\circ} \mathrm{C}, \mathrm{pH}\left(\mathrm{H}_{2} \mathrm{O}\right)=6.2 \\
40 \% \text { water content } \\
\text { total } \mathrm{C}=1.2 \% \text {, total } \\
\mathrm{N}=0.11 \%\end{array}$ & Weight loss & 50 & 10 & [59] \\
\hline & & & $\begin{array}{l}40^{\circ} \mathrm{C} \text {, total } \mathrm{C}=1.2 \% \\
\text { total } \mathrm{N}=0.11 \%, \mathrm{pH} \\
\left(\mathrm{H}_{2} \mathrm{O}\right)=6.2,40 \% \\
\text { water content }\end{array}$ & & 40 & 17 & \\
\hline PHB & Films & Soil & $\begin{array}{l}28^{\circ} \mathrm{C} \\
37^{\circ} \mathrm{C} \\
60^{\circ} \mathrm{C}\end{array}$ & Weight loss & 57.386 .725 .9 & 56 & [78] \\
\hline \multirow[t]{2}{*}{$\begin{array}{l}\text { PHBV (HV of } \\
11 \%)\end{array}$} & Films & $\begin{array}{l}1: 1 \text { mixture of } \\
\text { black soil and } \\
\text { leaf mold for } \\
\text { gardening }\end{array}$ & $25-30^{\circ} \mathrm{C}$ & Weight loss & $\sim 28$ & 180 & [79] \\
\hline & & & & & $\sim 48$ & 90 & \\
\hline \multicolumn{8}{|l|}{$\begin{array}{l}\text { PHBV/untreated } \\
\text { abaca fiber }\end{array}$} \\
\hline $\begin{array}{l}\text { PHBV/Aa-abaca } \\
\text { fiber }\end{array}$ & & & & & $\sim 48$ & 180 & \\
\hline $\begin{array}{l}\text { PHBV } 6.2 \text { mol } \\
\% \text { HV content }\end{array}$ & Films & $\begin{array}{l}\text { Garden soil } \\
270.4 \mathrm{~g} / \mathrm{kg} \text { of } \\
\text { organic matter, } \\
35.6 \% \\
\text { humidity and } \\
\mathrm{pH}\left(\mathrm{CaCl}_{2}\right) 5.1\end{array}$ & $23^{\circ} \mathrm{C}$ & Weight loss & 100 & 30 & [53] \\
\hline
\end{tabular}


Table 1 (continued)

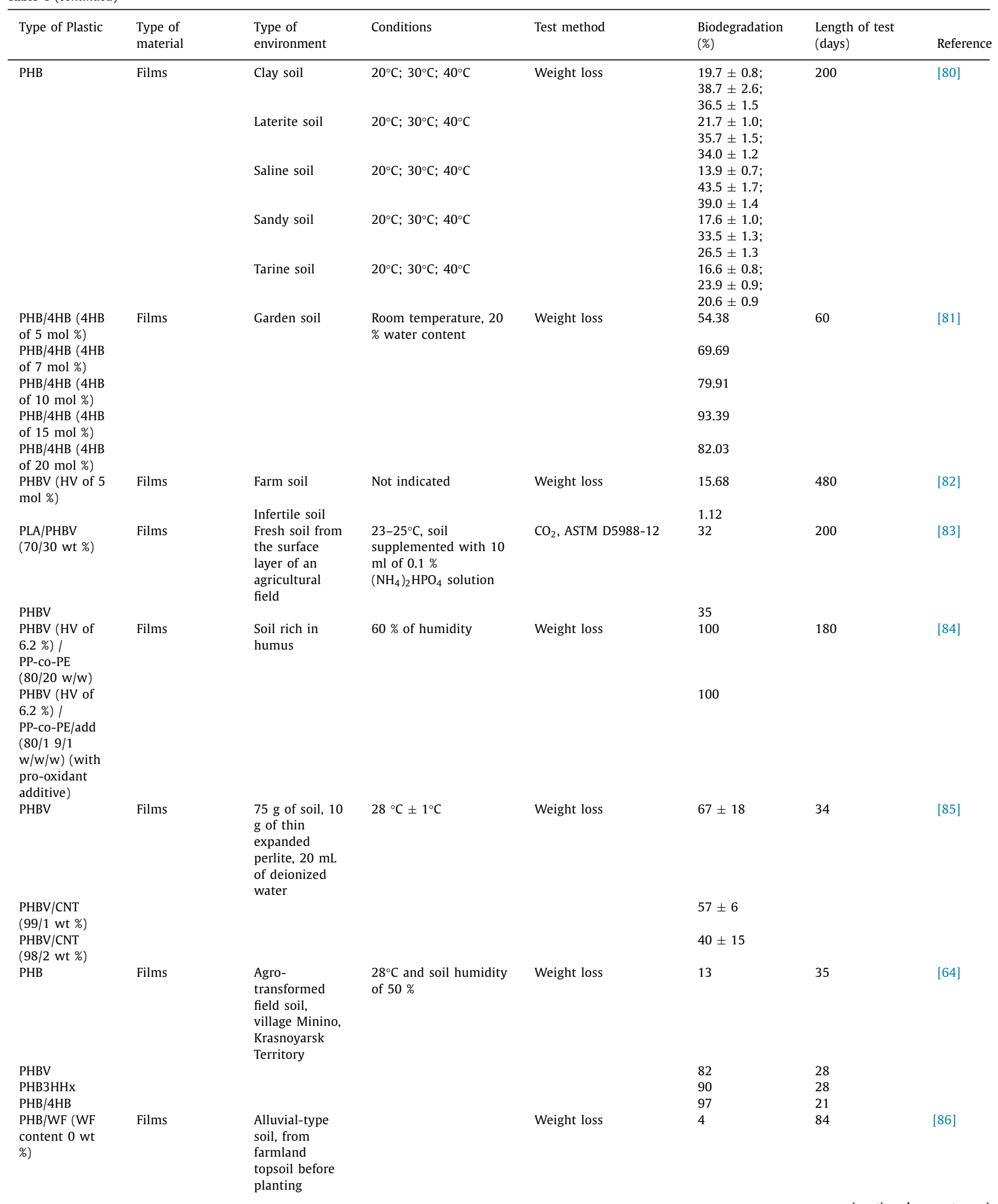


Table 1 (continued)

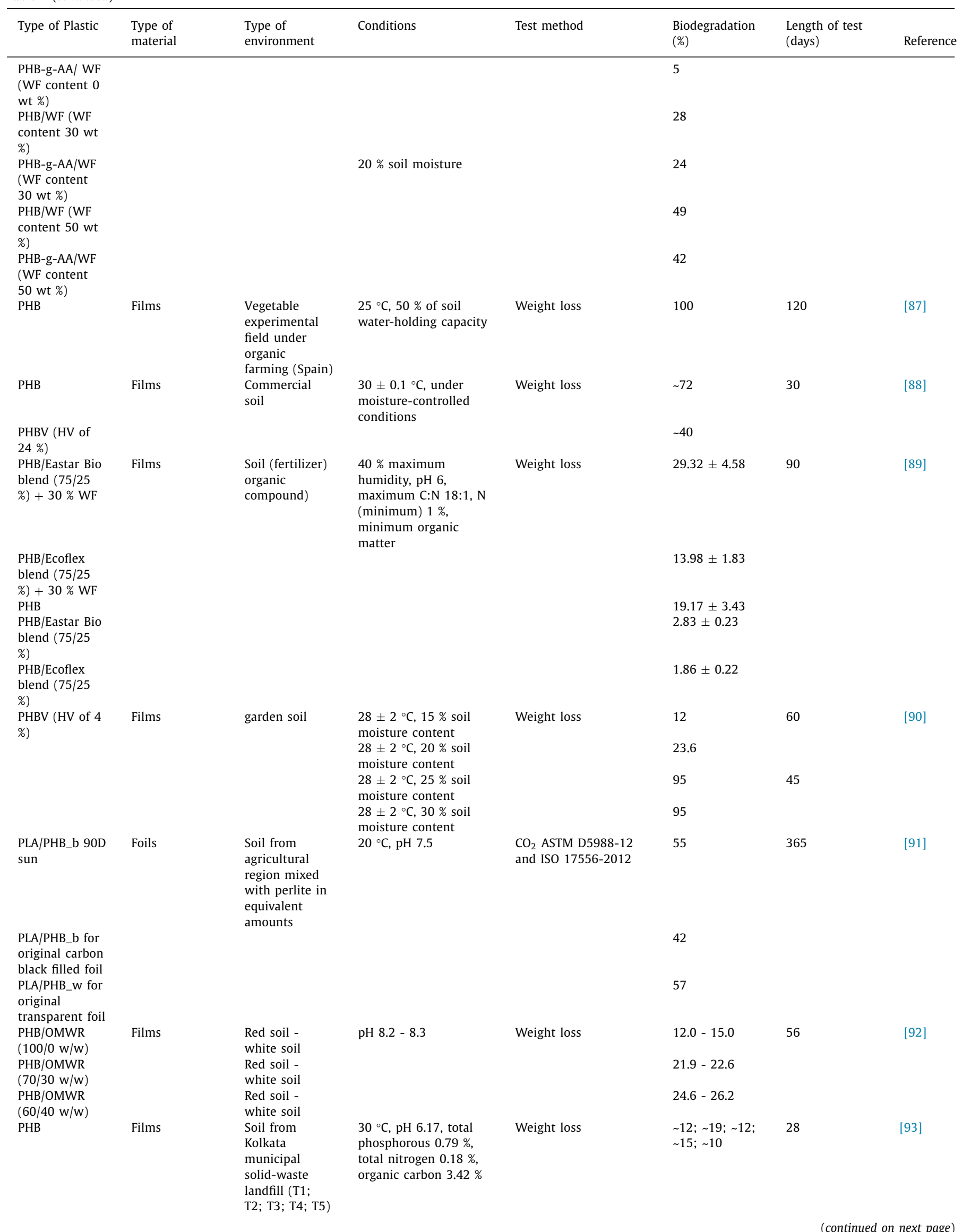


Table 1 (continued)

\begin{tabular}{|c|c|c|c|c|c|c|c|}
\hline Type of Plastic & $\begin{array}{l}\text { Type of } \\
\text { material }\end{array}$ & $\begin{array}{l}\text { Type of } \\
\text { environment }\end{array}$ & Conditions & Test method & $\begin{array}{l}\text { Biodegradation } \\
(\%)\end{array}$ & $\begin{array}{l}\text { Length of test } \\
\text { (days) }\end{array}$ & Reference \\
\hline PHB & Films & Paleudult soil & $\mathrm{pH} 6.17$ & Weight loss & 100 & 180 & {$[94]$} \\
\hline РНВ3НHx & Films & $\begin{array}{l}\text { Soil composted } \\
\text { in the farm of } \\
\text { Chubu } \\
\text { University }\end{array}$ & $\begin{array}{l}34{ }^{\circ} \mathrm{C} \text {, pH } 5.3 \text {, relative } \\
\text { humidity of } 90 \%\end{array}$ & Weight loss & $1.91-7.41$ & 28 & [95] \\
\hline PHA & Films & $\begin{array}{l}\text { Alluvial-type } \\
\text { soil obtained } \\
\text { from Taiwan } \\
\text { Kaohsiung } \\
\text { farmland } \\
\text { topsoil }\end{array}$ & $30-40 \%$ soil moisture & Weight loss & 25 & 60 & [96] \\
\hline PHA-g-MA & & & & & $\sim 30$ & & \\
\hline $\begin{array}{l}\text { PHA-g- } \\
\text { MA/TPLF ( } 20 \% \\
\text { w/w) }\end{array}$ & & & & & $\sim 65$ & & \\
\hline $\begin{array}{l}\text { PHA-g-MA/PF } \\
(20 \% \mathrm{w} / \mathrm{w})\end{array}$ & & & & & $\sim 70$ & & \\
\hline $\begin{array}{l}\text { PHA-g- } \\
\text { MA/TPLF (40 \% } \\
\text { w/w) }\end{array}$ & & & & & $\sim 85$ & & \\
\hline $\begin{array}{l}\text { PHA-g-MA/PF } \\
(40 \% \mathrm{w} / \mathrm{w})\end{array}$ & & & & & $\sim 90$ & & \\
\hline PHA & Films & $\begin{array}{l}\text { Alluvial soil } \\
\text { obtained from } \\
\text { farmland } \\
\text { topsoil before } \\
\text { planting }\end{array}$ & $35 \%$ soil moisture & Weight loss & 33 & 140 & [97] \\
\hline PHA-g-MA & & & & & 35 & & \\
\hline $\begin{array}{l}\text { PHA-g-MA/TPF } \\
(20 \% \mathrm{w} / \mathrm{w})\end{array}$ & & & & & 75 & & \\
\hline $\begin{array}{l}\text { PHA-g-MA/t - } \\
\text { TPF }(20 \% \mathrm{w} / \mathrm{w})\end{array}$ & & & & & 66 & & \\
\hline $\begin{array}{l}\text { PHA-g-MA/TPF } \\
(40 \% \mathrm{w} / \mathrm{w})\end{array}$ & & & & & 88 & & \\
\hline $\begin{array}{l}\text { PHA-g-MA/t- } \\
\text { TPF ( } 40 \% \\
\text { w/w) }\end{array}$ & & & & & 84 & & \\
\hline PHA & Powder & $\begin{array}{l}\text { Natural soils, } \\
\text { collected from } \\
\text { the surface } \\
\text { layer of one } \\
\text { field and two } \\
\text { forests or soil } \\
\text { ( } 15 \text { g, plough } \\
\text { layer, haplic } \\
\text { chernozem; } \\
\text { dry weight } \\
88.4 \% \text {; soil } \\
\text { texture, silty } \\
\text { loam, volatile } \\
\text { solids } 5.55 \% \text {; } \\
\text { soil organic } \\
\text { matter } 3.05 \% \text { ) }\end{array}$ & $25^{\circ} \mathrm{C}$ & $\mathrm{CO}_{2}$ ISO 17556 (2019) & $85.8-96.4$ & $150-170$ & [57] \\
\hline & & & $37{ }^{\circ} \mathrm{C}$ & & $71.1-93.0$ & $90-170$ & \\
\hline PHB & Films & $\begin{array}{l}\text { Artificial soil } \\
\text { according to } \\
\text { ASTM G } \\
\text { 160-03 }\end{array}$ & ASTM G 160-03 & Weight loss & $17.8 \pm 0.64$ & 86 & [98] \\
\hline $\begin{array}{l}\text { PHB/PP-g- } \\
\text { MA/clay (92 \% } \\
\text { | } 5 \% \text { / } 3 \% \text { ) }\end{array}$ & & & & & $22.5 \pm 0.24$ & & \\
\hline $\begin{array}{l}\text { PHB/PP-g- } \\
\text { MA/clay (94.5 } \\
\% \text { / } 2.5 \% \text { / } 3 \%)\end{array}$ & & & & & $25.9 \pm 0.67$ & & \\
\hline PHBV & Films & $\begin{array}{l}\text { Red clay } \\
\text { latosol soil } \\
\text { from a } 0 \text { to } 15 \\
\text { cm depth } \\
\text { profile }\end{array}$ & $\begin{array}{l}28^{\circ} \mathrm{C}, 60 \% \text { of the } \\
\text { moisture capacity }\end{array}$ & Weight loss & 10 & 28 & [99] \\
\hline
\end{tabular}


Table 1 (continued)

\begin{tabular}{|c|c|c|c|c|c|c|c|}
\hline Type of Plastic & $\begin{array}{l}\text { Type of } \\
\text { material }\end{array}$ & $\begin{array}{l}\text { Type of } \\
\text { environment }\end{array}$ & Conditions & Test method & $\begin{array}{l}\text { Biodegradation } \\
(\%)\end{array}$ & $\begin{array}{l}\text { Length of test } \\
\text { (days) }\end{array}$ & Reference \\
\hline PHBV-AgNP & & & & & 8 & & \\
\hline \multirow[t]{5}{*}{ PHB } & Films & $\begin{array}{l}\text { Natural soil } \\
\text { from } \\
\text { agricultural } \\
\text { land of the } \\
\text { clay-loam type } \\
\text { (clay } 29 \% \text {, silt } \\
28 \% \text {, and sand } \\
43 \% \text { ) }\end{array}$ & $\begin{array}{l}25^{\circ} \mathrm{C}, \mathrm{C}: \mathrm{N}=8, \mathrm{pH} 7.9 \text {, } \\
\text { water holding capacity } \\
80 \% \text {, total nitrogen } 2 \\
\% \text {, organic carbon } 0.13 \\
\%\end{array}$ & $\mathrm{CO}_{2}$, ISO 175562019 & $\sim 88 \sim 97$ & 120360 & [49] \\
\hline & Powder & $\begin{array}{l}\text { Natural soil } \\
\text { mixture from } \\
\text { three sources: } \\
\text { sandy and } \\
\text { forest }\end{array}$ & $\begin{array}{l}25^{\circ} \mathrm{C}, \mathrm{C}: \mathrm{N}=8, \mathrm{pH} 7.9, \\
\text { water holding capacity } \\
53 \% \text {, total nitrogen } 7.9 \\
\% \text {, organic carbon } 0.51 \\
\%\end{array}$ & & $\sim 90$ & 120 & \\
\hline & & $\begin{array}{l}\text { Standard soil: } \\
\text { Prepared } \\
\text { according to } \\
\text { ISO } \\
17556: 2012\end{array}$ & $\begin{array}{l}25^{\circ} \mathrm{C}, \mathrm{C}: \mathrm{N}=10, \mathrm{pH} \\
8.4 \text {, water holding } \\
\text { capacity } 50 \% \text {, total } \\
\text { nitrogen } 3.7 \% \text {, organic } \\
\text { carbon } 0.22 \%\end{array}$ & & $\sim 86$ & & \\
\hline & & $\begin{array}{l}\text { Natural soil, } \\
\text { mixture from } \\
\text { three sources: } \\
\text { sandy and } \\
\text { forest }\end{array}$ & $\begin{array}{l}25^{\circ} \mathrm{C}, \mathrm{C}: \mathrm{N}=11, \mathrm{pH} \\
7.4 \text {, water holding } \\
\text { capacity } 80 \% \text {, total } \\
\text { nitrogen } 6.8 \% \text {, organic } \\
\text { carbon } 0.31 \%\end{array}$ & & $\sim 95$ & & \\
\hline & & $\begin{array}{l}\text { Natural soil: } \\
\text { Sandy loam } \\
\text { (Sand } 62 \% \text {, Silt } \\
27 \% \text { ) }\end{array}$ & $\begin{array}{l}28^{\circ} \mathrm{C}, \mathrm{C}: \mathrm{N}=52, \mathrm{pH} \\
7.6 \text {, water holding } \\
\text { capacity } 60 \% \text {, total } \\
\text { nitrogen } 4.2 \% \text {, organic } \\
\text { carbon } 0.03 \%\end{array}$ & & $\sim 98$ & & \\
\hline
\end{tabular}

Abbreviations: Wood flour (WF), 4-hydroxybutyrate (4HB), 3-hydroxyhexanoate (3HHx), olive pomace stone-rich fraction (SF), olive pomace pulp-rich fraction (PF) olive

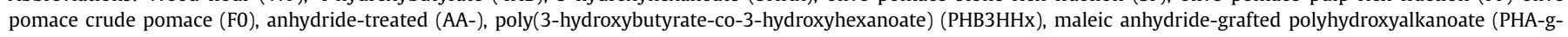
MA), PHA-g-AA, coupling agent-treated palm fibre (TPLF), palm fibre (PF), tea plant fibre (TPF) treated (crosslinked) tea plant fibre (t - TPF), carbon nanotubes (CNT), cel-

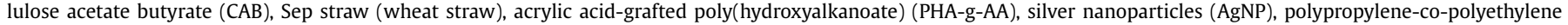

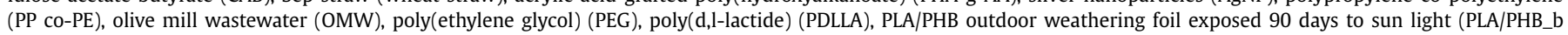
90D), graphitized polypropylene with maleic anhydride (PP-g-MA), PHB-g-acrylic acid PHB-g-AA), acrylic acid-grafted poly(hydroxyalkanoate) (PHA-g-AA).

\section{PHA field tests}

The most significant weakness of the existing standards for testing biodegradation of plastics in the soil is their weak reproducibility [47]. Several factors, including the soil type and biodiversity, the conditions such as temperature, water content, or $\mathrm{pH}$, and the measuring method may affect the reproducibility of the results [47]. For example, if the plastic is the only carbon source available for microbial growth, the test may present a higher rate of biodegradation compared to an environment where other carbon sources, and easier to biodegrade, are available. Testing the biodegradation of plastics in real environmental conditions is essential to understand the impact of plastics pollution better.

Changes in soil temperature, humidity, and even microbial composition can lead to different PHA biodegradation rates [50]. In a study conducted in Vietnamese soil, and contradictory to most of the results herein presented, PHB (evaluated by weight loss) degraded better than PHBV [100]. The authors justified these results with the differences in the composition of soil microbial communities and in the diversity of their PHA depolymerases [80]. In this study, the considerable decrease in the molecular weight and the increase in PHA polydispersity indicated that the polymers were cleaved, originating minor fragments with different polymerization degrees.

The shape of the films, soil $\mathrm{pH}$, soil water content, and moisture content of the polymer can also influence the PHA biodegradation rate. PHA films were found to be degraded at a faster rate than polymer pellets due to their higher surface area, which permitted a better microbial attachment and faster biofilms formation [100]. In this study, at weakly acidic pH (e.g., 5.48) the PHA biodegradation rate was higher than the biodegradation rate registered in an- other soil with a pH value close to neutrality (6.63), which may be linked to differences in the microbial diversity and activity. Soil pH can indeed determine microbial activity, since some species have narrow $\mathrm{pH}$ tolerance and cannot survive in acidic environments, and also because $\mathrm{pH}$ may influence the availability of soil nutrients (e.g., ammonia and nitrate), which are essential to certain microbial species [101]. However, a weakly acidic soil is a favourable condition for the development of fungi, that were the major PHA degraders in this soil, while bacteria were dominant in a soil with a neutral $\mathrm{pH}$ value [100].

Some microorganisms have enzymes with broad substrate specificity and can degrade both scl-PHA and mcl-PHA. However, most PHA-degrading microorganisms produce enzymes specific for a particular type of PHA substrate [102]. Thus, the same polymers in different soils may present different biodegradation rates [103]. The water content affected the PHB biodegradation which seems to be favoured in soils with higher water content [104]. The water absorption capacity of the polymer seems to influence biodegradation. Indeed, $\mathrm{PHB} 3 \mathrm{HHx} / \mathrm{KF}$ composites (which have higher water absorption capacity than $\mathrm{PHB} 3 \mathrm{HHx}$ ) presented a higher percentage of biodegradation (determined by weight loss) than $\mathrm{PHB} 3 \mathrm{HHx}$ [104].

Another factor affecting the biodegradation rate of PHA films in soils is whether they are buried or not. PHA films on the sediment surface are degraded at a slower rate than those buried in the sediment [62]. The biodegradation rate (evaluated by weight loss) of PHB films placed on the soil surface was $50 \%$ slower than when the PHB was buried [105]. Buried films are surrounded by soil, and thus the exposed surface area for microbial attack is higher, which results in higher biodegradation rates [62]. Moreover, the microbial communities colonizing buried, and non-buried films are different 
(e.g., buried films are in contact with both aerobic and anaerobic bacteria), which may also influence the biodegradation rates [62].

Light, especially UV, was found to influence the biodegradation process. With UV pre-treatment, PHB and its films degraded faster than the PHB films untreated [106]. The UV treatment damaged the films creating increased cracks, which accelerated the biodegradation process [106]. The techniques used to produce the films may also influence the biodegradation rate due to changes in their three-dimensional structures and surface area, which can benefit or hamper the microbial colonization of the polymeric films [106]. For example, PHB nanofiber films prepared by an electrospinning technique presented higher weight loss due to the three-dimensional structures and large surface area of nanofibers compared to the PHB produced with the solvent-cast technique [106].

\section{PHA blends biodegradation in soil}

The cost of bio-based plastics is still higher than that of the plastics produced from petroleum raw materials. The blending of PHAs with other bioplastics or with naturally decomposable materials, such as agricultural waste and natural fibers, is a strategy to reduce production cost or change the properties according to the goals established [32]. This process also changes the biodegradation properties and requires a new assessment of the blends biodegradability. Some blends can be used to change the properties of PHA, but these blends can also change the biodegradation behaviour of PHA, increasing or decreasing the biodegradation rate, depending on the material blended to the PHA [62,65,70,86,104113]. These different effects can be the result of 1) an overall crystallinity change since crystalline zones are less accessible for the microorganisms, 2) microstructural defects that facilitate the adhesion of bacteria, or even to 3 ) increased hydrophilicity of the blends that facilities water adsorption that is fundamental for the microorganism biodegradation [65,73].

The biodegradation behaviours of the PHB/lignin blends (films) were analysed in a soil field study. The results indicated that PHB films disintegrated with $45 \%$ of weight loss within 12 months, however, the PHB/lignin blends had only a weight loss of $12 \%$ when $10 \%$ of lignin was present. These results suggest that the presence of lignin can reduce the PHB biodegradation, probably by hampering the colonization by microorganisms (most likely due to its hydrophobicity), which improved the resistance of the blends to microbial activity [114].

Jeszeova et al. [91] tested PLA/PHB foils with the ASTM D5988 biodegradation test $\left(\mathrm{CO}_{2}\right.$ production), and the PLA/PHB white foils showed the best biodegradation ( $57 \%$ ), followed by the PLA/PHB black foils previous exposed to outdoor weathering for 90 days (55\%) and finally by the PLA/PHB black foils (42\%) (Table 1). The microorganisms present in the soil, and potentially involved in the biodegradation of the films, were identified by using culturedependent methods (i.e., microbial strains were cultivated in three different growth media containing PLA/PHB blend, PHB or PLA) and culture-independent methods (i.e., 16S rRNA gene analysis by denaturing gradient gel electrophoresis (DGGE) and cloning). This strategy allowed the identification and isolation of several PLA/PHB blend degrading microorganisms assigned to several genera, including Bacillus, Streptomyces, Rhodococcus, Saccharothrix, Fusarium, Trichoderma, and Penicillium. This approach can be useful to get insights into the biodegradation process because the microorganisms are the main responsible for the biodegradation of the plastics.

The biodegradation of PHBV/wood flour (WF) was more significant in the laboratory (PHA50WF -35\%, PHA20WF - $36 \%$ ) using ASTM D5988 than in the filed study. Chan et al. [67] explained this result due to the higher moisture content of the soil laboratory test, which has been shown to accelerate the biodegrada- tion of both wood and PHA. But contradictory results demonstrating higher biodegradation of PHB in garden soil than in laboratory settings (using the same soil) have also been reported [71].

As previously indicated, the temperature can significantly influence the biodegradation process and PLA/PHA mulches degraded more extensively in the soil during the summer than in wintertime because the warmer temperatures promoted microbial activities [115].

In conclusion: the rate of biodegradation in soil is influenced by several factors, including the properties of the PHA material such as crystallinity, surface area, type of PHA, composition, and shape, the environmental factors such as temperature, moisture level, $\mathrm{pH}$, and nutrient supply, the microbial communities and the activity and specificity of microbial depolymerases. These factors interact, creating different soil environments and different biodegradation potentials from place to place, from season to season.

\section{Microorganisms involved in PHA biodegradation}

Microorganisms are the main responsible for the biodegradation of PHA-based plastics in several ecosystems. Biodegradation depends on the existence of PHA degrading enzymes (PHA depolymerases) produced by microorganisms that can hydrolyse waterinsoluble PHA into water-soluble forms, so it can be used by these microorganisms [116]. In soil, differences in the rate of PHA biodegradation can be due to several factors, including the composition of the microbial communities and to type and specificity of the depolymerases that they produce [31].

Almost 600 PHA depolymerases with different substrate specificities have been identified in various microorganisms [117]. Among intracellular and extracellular depolymerases and through the analysis of their sequences, they were classified into $8 \mathrm{su}$ perfamilies and in 38 homologous families [117]. Several bacteria capable of PHA biodegradation are assigned to genera: Stenotrophomonas, Alcaligenes, Comamonas, Rhodococcus, Rhodocyclus, Variovorax, Acinetobacter, Pseudomonas, Bacillus, Xanthomonas, Syntrophomonas, Ilyobacter, and Ralstonia [36,50,118].

In several studies, fungi were able to biodegrade PHA polymers and were the dominant microorganisms colonizing the surface of the polymer [57,119]. Fungi have higher biodegradation capability compared to bacteria because their PHA-depolymerases have higher mobility [120]. Several groups of fungi, including ascomycetes, basidiomycetes, deuteromycetes (e.g., Penicillium simplicissimum) zygomycetes, and micromycetes (e.g., Penicillium, Paecilomyces, Acremonium, Verticillium, and Zygosporium), among others, have been identified as capable of degrading PHA polymers [50,121].

A rough surface allows better adhesion of the microorganisms and water, which accelerates the biodegradation of the polymers. A smooth surface decreases adhesion, which delays the biodegradation process [114]. The lag phase preceding the biodegradation of PHA films in soil may take days, weeks, or even months, depending on the composition and shape of the PHA and on the environmental conditions. This lag period is the time needed for microbial adhesion to the material's surface and for expression and release of extracellular depolymerases [31]. Usually, a higher degree of crystallinity decreases microbial degradation, while the amorphous regions are easily degraded [122]. In analogous ecosystems, in different regions, the biodegradability of PHBV is related to the numbers of PHBV degraders and is dependent on the growth conditions for PHBV degraders $[83,123,124]$. Soil microcosms with higher functional diversity present better PHA biodegradation capacity than soil microcosms with lower functional diversity [94,103].

It has been verified that the percentage of true PHA-degrading microorganisms is relatively low in the microbial community colonizing the surface of the polymer $[50,64]$. Other types of microor- 
ganisms (commensal organisms) may develop on the surface by growing on intermediate compounds of PHA biodegradation, such as oligomers, monomers, acetoacetate, and other compounds produced by the true PHA degraders [31]. The isolation of the PHA degraders from the non-PHA degraders can be accomplished by collecting the microbial biofilms developed on the surface of the polymers, followed by cultivation on typical microbiological media containing PHA as the unique carbon source. This strategy is useful and widely used and is designated by the "clear zone technique". Clear zones around the colonies of microorganisms with PHAdepolymerase activity will develop and indicate PHA biodegradation $[31,102,125]$. The microorganisms forming theses colonies can be further cultivated and identified.

Interestingly, some microorganisms can degrade several types of PHA in soil, but others can only degrade a specific PHA type. Volova et al. [31] use the clear zone technique together with molecular-genetic methods (rRNA gene sequence) and found that PHB was degraded by bacteria of the genera Mitsuaria, Chitinophaga, and Acidovorax, but they were not detected on the surface of the copolymers $\mathrm{PHB} / 4 \mathrm{HB}$, PHB3HHx, and PHBV. Roseateles depolymerans, Streptomyces gardneri, and Cupriavidus $s p$. were specific degraders of $\mathrm{PHB} / 4 \mathrm{HB}$, Roseomonas massiliae, and Delftia acidovorans degraded PHBV, and Pseudoxanthomonas sp., Pseudomonas fluorescens, Ensifer adhaerens, and Bacillus pumilus degraded specifically PHB3HHx [31]. Streptomyces were capable of degrading all PHA polymers [31]. Some microorganisms can produce several types of depolymerases, and thus have a broader range of PHA biodegradation potential, while others only produce one kind of depolymerase capable of PHA biodegradation [102].

\section{Conclusion}

Several factors can influence the PHAs biodegradation in soil, including environmental conditions, the properties of the materials, and the presence of PHA-degrading microorganisms, which are the key factor to achieve biodegradation. The blend of PHAs with other types of materials makes these polymers more competitive and allows their application in several areas. However, testing and validating the biodegradation of those new blends is necessary to determine if they meet the environmental requirements. In order to understand the biodegradation process in soil, it is essential to conduct laboratory and field tests in different conditions and control or evaluate all the factors that can influence the process. It is also fundamental to identify the microorganisms and the enzymes in those environments since they have different specificities and biodegradation capabilities.

\section{Declaration of Competing Interest}

The authors declare that they have no known competing financial interests or personal relationships that could have appeared to influence the work reported in this paper.

\section{Acknowledgement}

Miguel Fernandes acknowledges the grant PD/BD/146195/2019 provided by the Portuguese Foundation for Science and Technology (FCT). This study was supported by the Portuguese Foundation for Science and Technology (FCT) under the scope of the strategic funding of UIDB/04469/2020 unit and BioTecNorte operation (NORTE-01-0145-FEDER-000004) funded by the European Regional Development Fund under the scope of Norte2020-Programa Operacional Regional do Norte.

\section{References}

[1] R. Jain, A. Tiwari, Biosynthesis of planet friendly bioplastics using renewable carbon source, J. Environ. Heal. Sci. Eng. 13 (2015) 11, doi:10.1186/ s40201-015-0165-3.

[2] D. Barnes, F. Galgani, R. Thompson, M. Barlaz, Accumulation and fragmentation of plastic debris in global environments, Philos. Trans. R. Soc. Lond. B. Biol. Sci. 364 (Aug.) (2009) 1985-1998, doi:10.1098/rstb.2008.0205.

[3] J.R. Jambeck, R. Geyer, C. Wilcox, T.R. Siegler, M. Perryman, A. Andrady, R. Narayan, K.L. Law, Plastic waste inputs from land into the ocean, Science 347 (Feb. 6223) (2015) 768LP-76771, doi:10.1126/science.1260352.

[4] J.H. Song, R.J. Murphy, R. Narayan, G.B.H. Davies, Biodegradable and compostable alternatives to conventional plastics, Philos. Trans. R. Soc. Lond. B. Biol. Sci. 364 (Jul. 1526) (2009) 2127-2139, doi:10.1098/rstb.2008.0289.

[5] E.F. Gómez, F.C. Michel, Biodegradability of conventional and bio-based plastics and natural fiber composites during composting, anaerobic digestion and long-term soil incubation, Polym. Degrad. Stab. 98 (12) (2013) 2583-2591 https://doi.org/10.1016/j.polymdegradstab.2013.09.018.

[6] X. Wang, A. Oehmen, E.B. Freitas, G. Carvalho, M.A.M. Reis, The link of feastphase dissolved oxygen (DO) with substrate competition and microbial selection in PHA production, Water Res. 112 (Apr.) (2017) 269-278, doi:10.1016/j. watres.2017.01.064.

[7] M.A.E.-H. El-Abd, H.H. El-Sheikh, S. Desouky, A. Shehab, Identification, Biodegradation and bio-evaluation of biopolymer produced from Bacillus thuringenesis, J. Appl. Pharm. Sci. 7 (Apr.) (2017) 103-110, doi:10.7324/JAPS. 2017.70414.

[8] M.V. Cruz, F. Freitas, A. Paiva, F. Mano, M. Dionísio, A.M. Ramos, M.A.M. Reis, Valorization of fatty acids-containing wastes and byproducts into short- and medium-chain length polyhydroxyalkanoates, N. Biotechnol. 33 (1) (2016) 206-215 https://doi.org/10.1016/j.nbt.2015.05.005.

[9] L. Shang, M. Jiang, H.N. Chang, Poly(3-hydroxybutyrate) synthesis in fed-batch culture of Ralstonia eutropha with phosphate limitation under different glucose concentrations, Biotechnol. Lett. 25 (Sep. 17) (2003) 1415-1419.

[10] P. Pavez, J.L. Castillo, C. González, M. Martínez, Poly- $\beta$-hydroxyalkanoate exert a protective effect against carbon starvation and frozen conditions in Sphingopyxis chilensis, Curr. Microbiol. (2009), doi:10.1007/s00284-009-9485-9.

[11] W.N. Chaudhry, N. Jamil, I. Ali, M.H. Ayaz, S. Hasnain, Screening for polyhydroxyalkanoate (PHA)-producing bacterial strains and comparison of PHA production from various inexpensive carbon sources, Ann. Microbiol. 61 (3) (2011) 623-629, doi:10.1007/s13213-010-0181-6.

[12] R. Ashby, D. Solaiman, Poly(hydroxyalkanoate) Biosynthesis from Crude Alaskan Pollock (Theragra chalcogramma) Oil, J. Polym. Environ. 16 (Oct.) (2008), doi:10.1007/s10924-008-0108-5.

[13] Y. Liu, Z. Zhan, H. Ye, X. Lin, Y. Yan, Y. Zhang, Accelerated biodegradation of PLA/PHB-blended nonwovens by a microbial community, RSC Adv. 9 (18) (2019) 10386-10394, doi:10.1039/C8RA10591J.

[14] J. Lu, R.C. Tappel, C.T. Nomura, Mini-review: biosynthesis of poly(hydroxyalkanoates), Polym. Rev. (2009), doi:10.1080/ 15583720903048243.

[15] A. Poli, P. Di Donato, G.R. Abbamondi, B. Nicolaus, Synthesis, production, and biotechnological applications of exopolysaccharides and polyhydroxyalkanoates by Archaea, Archaea (2011) 13, doi:10.1155/2011/693253.

[16] M.R. López-Cuellar, J. Alba-Flores, J.N.G. Rodríguez, F. Pérez-Guevara, Production of polyhydroxyalkanoates (PHAs) with canola oil as carbon source, Int. J. Biol. Macromol. 48 (1) (2011) 74-80 https://doi.org/10.1016/j.ijbiomac.2010. 09.016.

[17] J. Han, J. Hou, H. Liu, S. Cai, B. Feng, J. Zhou, H. Xiang, Wide distribution among halophilic Archaea of a novel polyhydroxyalkanoate synthase subtype with homology to bacterial type III synthases, Appl. Environ. Microbiol. 76 (Oct.) (2010) 7811-7819, doi:10.1128/AEM.01117-10.

[18] A. Steinbüchel, T. Lütke-Eversloh, Metabolic engineering and pathway construction for biotechnological production of relevant polyhydroxyalkanoates in microorganisms, Biochem. Eng. J. 16 (2) (2003) 81-96 https://doi.org/10. 1016/S1369-703X(03)00036-6.

[19] M. Zinn, R. Hany, Tailored material properties of polyhydroxyalkanoates through biosynthesis and chemical modification, Adv. Eng. Mater. 7 (May) (2005) 408-411, doi:10.1002/adem.200500053.

[20] A. Santhanam, S. Sasidharan, Microbial production of polyhydroxy alkanotes (PHA) from Alcaligens spp. and Pseudomonas oleovorans using different carbon sources, Afr. J. Biotechnol. 9 (May) (2010) 3144-3150.

[21] E. Conte, V. Catara, S. Greco, M. Russo, R. Alicata, L. Strano, A. Lombardo, S. Di Silvestro, A. Catara, Regulation of polyhydroxyalkanoate synthases (phaC1 and phaC2) gene expression in Pseudomonas corrugata, Appl. Microbiol. Biotechnol. 72 (Oct. 5) (2006) 1054-1062, doi:10.1007/s00253-006-0373-y.

[22] T. Yamane, X.-F. Chen, S. Ueda, Polyhydroxyalkanoate synthesis from alcohols during the growth of Paracoccus denitrificans, FEMS Microbiol. Lett. 135 (Jan. 2-3) (1996) 207-211, doi:10.1111/j.1574-6968.1996.tb07991.x.

[23] M. Koller, A. Atlic, M. De Sousa Dias, A. Reiterer, G. Braunegg, Microbial PHA production from waste raw materials, in: G.-Q. Chen (Ed.), Plastics from Bacteria: Natural Functions and Applications, 14, Springer, 2009, pp. 85-119.

[24] C. Morais, F. Freitas, M.V Cruz, A. Paiva, M. Dionísio, M.A.M. Reis, Conversion of fat-containing waste from the margarine manufacturing process into bacterial polyhydroxyalkanoates, Int. J. Biol. Macromol. 71 (2014) 68-73 https: //doi.org/10.1016/j.ijbiomac.2014.04.044. 
[25] K. Tanaka, K. Katamune, A. Ishizaki, Fermentative production of $\operatorname{poly}(\beta$ hydroxybutyric acid) from xylose via L-lactate by a two-stage culture method employing Lactococcus lactis IO-1 and Alcaligenes eutrophus, Can. J. Microbiol. 41 (Dec. 13) (1995) 257-261, doi:10.1139/m95-194.

[26] J.A. Ramsay, E. Berger, R. Voyer, C. Chavarie, B.A. Ramsay, Extraction of poly3-hydroxybutyrate using chlorinated solvents, Biotechnol. Tech. 8 (8) (1994) 589-594, doi:10.1007/BF00152152.

[27] X. Jiang, J.A. Ramsay, B.A. Ramsay, Acetone extraction of mcl-PHA from Pseudomonas putida KT2440, J. Microbiol. Methods 67 (2) (2006) 212-219 https://doi.org/10.1016/j.mimet.2006.03.015.

[28] E. Berger, B.A. Ramsay, J.A. Ramsay, C. Chavarie, G. Braunegg, PHB recovery by hypochlorite digestion of non-PHB biomass, Biotechnol. Tech. 3 (4) (1989) 227-232, doi:10.1007/BF01876053.

[29] F.M. Kapritchkoff, A.P. Viotti, R.C.P. Alli, M. Zuccolo, J.G.C. Pradella, A.E. Maiorano, E.A. Miranda, A. Bonomi, Enzymatic recovery and purification of polyhydroxybutyrate produced by Ralstonia eutropha, J. Biotechnol. 122 (Apr. 4) (2006) 453-462, doi:10.1016/j.jbiotec.2005.09.009.

[30] G.J.M. de Koning, M. Kellerhals, C. van Meurs, B. Witholt, A process for the recovery of poly(hydroxyalkanoates) from Pseudomonads part 2: process development and economic evaluation, Bioprocess Eng. 17 (1) (1997) 15-21, doi:10.1007/s004490050346.

[31] T.G. Volova, S.V Prudnikova, O.N. Vinogradova, D.A. Syrvacheva, E.I. Shishatskaya, Microbial degradation of polyhydroxyalkanoates with different chemical compositions and their biodegradability, Microb. Ecol. 73 (Feb. 2) (2017) 353-367, doi:10.1007/s00248-016-0852-3.

[32] A. Shrivastav, S. Mishra, I. Pancha, D. Jain, S. Bhattacharya, S. Patel, S. Mishra, Biodegradability studies of polyhydroxyalkanoate (PHA) film produced by a marine bacteria using Jatropha biodiesel byproduct as a substrate, World J. Microbiol. Biotechnol. 27 (Jul.) (2011), doi:10.1007/s11274-010-0605-2.

[33] S.Y. Lee, J. Choi, Production and degradation of polyhydroxyalkanoates in waste environment, Waste Manage. 19 (2) (1999) 133-139 https://doi.org/10. 1016/S0956-053X(99)00005-7.

[34] M. Shimao, Biodegradation of plastics, Curr. Opin. Biotechnol. 12 (3) (2001) 242-247 https://doi.org/10.1016/S0958-1669(00)00206-8.

[35] G.G. Choi, H.W. Kim, Y.H. Rhee, Enzymatic and non-enzymatic degradation of poly (3-hydroxybutyrate-co-3-hydroxyvalerate) copolyesters produced by $\mathrm{Al}$ caligenes sp. MT-16, J. Microbiol. 42 (Dec. 4) (2004) 346-352.

[36] J. Mergaert, J. Swings, Biodiversity of microorganisms that degrade bacterial and synthetic polyesters, J. Ind. Microbiol. 17 (5) (1996) 463-469, doi:10.1007/ BF01574777.

[37] E.A. Dawes, Polyhydroxybutyrate: an intriguing biopolymer, Biosci. Rep. 8 (Dec. 6) (1988) 537-547, doi:10.1007/bf01117332.

[38] C.-S. Ha, W.-J. Cho, Miscibility, properties, and biodegradability of microbial polyester containing blends, Prog. Polym. Sci. 27 (4) (2002) 759-809 https: //doi.org/10.1016/S0079-6700(01)00050-8.

[39] M. Pietrini, L. Roes, M.K. Patel, E. Chiellini, Comparative life cycle studies on poly(3-hydroxybutyrate)-based composites as potential replacement for conventional petrochemical plastics, Biomacromolecules 8 (Jul. 7) (2007) 22102218, doi:10.1021/bm0700892.

[40] D.Z. Bucci, L.B.B. Tavares, I. Sell, PHB packaging for the storage of food products, Polym. Test. 24 (5) (2005) 564-571 https://doi.org/10.1016/j. polymertesting.2005.02.008.

[41] G.-Q. Chen, Q. Wu, The application of polyhydroxyalkanoates as tissue engineering materials, Biomaterials 26 (33) (2005) 6565-6578 https://doi.org/10. 1016/j.biomaterials.2005.04.036.

[42] ISO 472. Plastics - Vocabulary, International Organization for Standardization, Geneva, Switzerland, 2013.

[43] A. D5988Standard Test Method for Determining Aerobic Biodegradation of Plastic Materials in Soil, ASTM International, West Conshohocken, PA, USA, 2018.

[44] I. 17556Plastics-Determination of the Ultimate Aerobic Biodegradability of Plastic Materials in Soil by Measuring the Oxygen Demand in a Respirometer or the Amount of Carbon Dioxide Evolved, International Organization for Standardization, Geneva, Switzerland, 2019

[45] NF U52-001Biodegradable Materials for Use in Agriculture and HorticultureMulching Products-Requirements and Test Methods, AFNOR Group, La Plaine St Denis, France, 2005.

[46] U. 11462Plastic Materials Biodegradable in Soil-Types, Requirements and Test Methods, Italian Organization for Standardization (UNI), 2012.

[47] D. Briassoulis, A. Mistriotis, Key parameters in testing biodegradation of biobased materials in soil, Chemosphere 207 (Sep.) (2018) 18-26, doi:10.1016/j. chemosphere.2018.05.024.

[48] E. 17033Plastics-Biodegradable Mulch Films for Use in Agriculture and Horticulture-Requirements and Test Methods, European Committee for Standardization, Brussels, Belgium, 2018.

[49] D. Briassoulis, A. Mistriotis, N. Mortier, M. Tosin, A horizontal test method for biodegradation in soil of bio-based and conventional plastics and lubricants, J. Clean. Prod. 242 (2020) 118392 https://doi.org/10.1016/j.jclepro.2019.118392.

[50] A.N. Boyandin, S.V Prudnikova, M.L. Filipenko, E.A. Khrapov, A.D. Vasil'ev, T.G. Volova, Biodegradation of polyhydroxyalkanoates by soil microbial communities of different structures and detection of PHA degrading microorganisms, Appl. Biochem. Microbiol. 48 (1) (2012) 28-36, doi:10.1134/ S0003683812010024.
[51] T. Suyama, Y. Tokiwa, P. Ouichanpagdee, T. Kanagawa, Y. Kamagata, Phylogenetic affiliation of soil bacteria that degrade aliphatic polyesters available commercially as biodegradable plastics, Appl. Environ. Microbiol. 64 (Dec. 12) (1998) 5008-5011.

[52] H. Nishida, Y. Tokiwa, Distribution of $\operatorname{poly}(\beta$-hydroxybutyrate) and $\operatorname{poly}(\varepsilon-$ caprolactone)aerobic degrading microorganisms in different environments, J. Environ. Polym. Degrad. 1 (3) (1993) 227-233, doi:10.1007/BF01458031.

[53] S.P.C. Gonçalves, S.M. Martins-Franchetti, D.L. Chinaglia, Biodegradation of the films of PP, PHBV and its blend in soil, J. Polym. Environ. 17 (4) (2009) 280, doi:10.1007/s10924-009-0150-y.

[54] J. Tao, C. Song, M. Cao, D. Hu, L. Liu, N. Liu, S. Wang, Thermal properties and degradability of poly(propylene carbonate)/poly( $\beta$-hydroxybutyrate-co$\beta$-hydroxyvalerate) (PPC/PHBV) blends, Polym. Degrad. Stab. 94 (4) (2009) 575-583 https://doi.org/10.1016/j.polymdegradstab.2009.01.017.

[55] L.V Lopez-Llorca, M.F. Colom Valiente, A. Gascon, A study of biodegradation of poly- $\beta$-hydroxyalkanoate (PHA) films in soil using scanning electron microscopy, Micron 24 (1) (1993) 23-29 https://doi.org/10.1016/0968-4328(93) 90012-P.

[56] C.A. Woolnough, L.H. Yee, T. Charlton, L.J.R. Foster, Environmental degradation and biofouling of 'green' plastics including short and medium chain length polyhydroxyalkanoates, Polym. Int. 59 (5) (2010) 658-667, doi:10.1002/ pi.2746.

[57] J. Šerá, L. Serbruyns, B. De Wilde, M. Koutný, Accelerated biodegradation testing of slowly degradable polyesters in soil, Polym. Degrad. Stab. 171 (2020) 109031 https://doi.org/10.1016/j.polymdegradstab.2019.109031.

[58] M.-N. Kim, A.-R. Lee, J.-S. Yoon, I.-J. Chin, Biodegradation of poly(3hydroxybutyrate), sky-green ${ }^{\circledR}$ and mater-bi ${ }^{\circledR}$ by fungi isolated from soils, Eur. Polym. J. 36 (Aug.) (2000) 1677-1685, doi:10.1016/S0014-3057(99)00219-0.

[59] H. Nishide, K. Toyota, M. Kimura, Effects of soil temperature and anaerobiosis on degradation of biodegradable plastics in soil and their degrading microorganisms, Soil Sci. Plant Nutr. 45 (Dec. 4) (1999) 963-972, doi:10.1080/ 00380768.1999 .10414346$.

[60] J. Mergaert, A. Webb, C. Anderson, A. Wouters, J. Swings, Microbial degradation of poly(3-hydroxybutyrate) and poly(3-hydroxybutyrate-co-3-hydroxyvalerate) in soils, Appl. Environ. Microbiol. 59 (Oct. 10) (1993) 3233-3238.

[61] J. Mergaert, C. Anderson, A. Wouters, J. Swings, K. Kersters, Biodegradation of polyhydroxyalkanoates, FEMS Microbiol. Lett. 103 (2) (1992) 317-321 https: //doi.org/10.1016/0378-1097(92)90325-I.

[62] N. Sridewi, K. Bhubalan, K. Sudesh, Degradation of commercially important polyhydroxyalkanoates in tropical mangrove ecosystem, Polym. Degrad. Stab. 91 (12) (2006) 2931-2940 https://doi.org/10.1016/j.polymdegradstab.2006.08. 027.

[63] Y.S. Salim, A. Sharon, S. Vigneswari, M.N. Mohamad Ibrahim, A.A. Amirul, Environmental degradation of microbial polyhydroxyalkanoates and oil palmbased composites, Appl. Biochem. Biotechnol. 167 (May 2) (2012) 314-326, doi:10.1007/s12010-012-9688-6.

[64] S.V. Prudnikova, O.N. Vinogradova, M.Y. Trusova, Specific character of bacterial biodegradation of polyhydroxyalkanoates with different chemical structure in soil, Dokl. Biochem. Biophys. (2017), doi:10.1134/S1607672917010185.

[65] L. Wei, S. Liang, A.G. McDonald, Thermophysical properties and biodegradation behavior of green composites made from polyhydroxybutyrate and potato peel waste fermentation residue, Ind. Crops Prod. 69 (2015) 91-103 https://doi.org/10.1016/j.indcrop.2015.02.011.

[66] M. Avella, E.G. La. Martuscelli, M. Raimo, P. Sadocco, G. Elegir, R. Riva, Poly(3hydroxybutyrate-co-3-hydroxyvalerate) and wheat straw fibre composites: thermal, mechanical properties and biodegradation behaviour, J. Mater. Sci. 35 (4) (2000) 829-836, doi:10.1023/A:1004773603516.

[67] C.-S. Wu, Preparation and characterization of polyhydroxyalkanoate bioplastic-based green renewable composites from rice husk, J. Polym. Environ. 22 (3) (2014) 384-392, doi:10.1007/s10924-014-0662-y.

[68] M.V. Arcos-Hernandez, B. Laycock, S. Pratt, B.C. Donose, M.A.L. Nikolić P. Luckman, A. Werker, P.A. Lant, Biodegradation in a soil environment of activated sludge derived polyhydroxyalkanoate (PHBV), Polym. Degrad. Stab. 97 (11) (2012) 2301-2312 https://doi.org/10.1016/j.polymdegradstab.2012.07.035.

[69] C.M. Chan, L.-J. Vandi, S. Pratt, P. Halley, D. Richardson, A. Werker, B. Laycock, Insights into the biodegradation of PHA/wood composites: micro- and macroscopic changes, Sustain. Mater. Technol. 21 (2019) e00099 https://doi.org/10. 1016/j.susmat.2019.e00099.

[70] S. Wang, P. Ma, R. Wang, S. Wang, Y. Zhang, Y. Zhang, Mechanical, thermal and degradation properties of poly(d,l-lactide)/poly(hydroxybutyrate-cohydroxyvalerate)/poly(ethylene glycol) blend, Polym. Degrad. Stab. 93 (7) (2008) 1364-1369 https://doi.org/10.1016/j.polymdegradstab.2008.03.026.

[71] P.C. Sabapathy, S. Devaraj, A. Parthipan, P. Kathirvel, Polyhydroxyalkanoate production from statistically optimized media using rice mill effluent as sustainable substrate with an analysis on the biopolymer's degradation potential, Int. J. Biol. Macromol. 126 (Apr.) (2019) 977-986, doi:10.1016/j.ijbiomac.2019. 01.003.

[72] S. Casarin, C. Rodrigues, O. Júnior, F. Rosário, J. Agnelli, Biodegradation in soil of the PHB/wood flour (80/20) and PHB/sisal fiber (80/20) tubes, Mater. Res. (Jan.) (2017), doi:10.1590/1980-5373-mr-2016-0904. 
[73] S. Lammi, E. Gastaldi, F. Gaubiac, H. Angellier-Coussy, How olive pomace can be valorized as fillers to tune the biodegradation of PHBV based composites, Polym. Degrad. Stab. 166 (2019) 325-333 https://doi.org/10.1016/j. polymdegradstab.2019.06.010.

[74] D. Erkske, I. Viskere, A. Dzene, V. Tupureina, L. Savenkova, Biobased polymer composites for films and coatings, Proc. Est. Acad. Sci. 55 (Jan.) (2006) 70-77.

[75] A.O. Pérez-Arauz, A.E. Aguilar-Rabiela, A. Vargas-Torres, A.-I. RodríguezHernández, N. Chavarría-Hernández, B. Vergara-Porras, M.R. López-Cuellar, Production and characterization of biodegradable films of a novel polyhydroxyalkanoate (PHA) synthesized from peanut oil, Food Packag. Shelf Life 20 (2019) 100297 https://doi.org/10.1016/j.fpsl.2019.01.001.

[76] S. Thomas, A.A. Shumilova, E.G. Kiselev, S.V. Baranovsky, A.D. Vasiliev, I.V. Nemtsev, A.P. Kuzmin, A.G. Sukovatyi, R.P. Avinash, T.G. Volova, Thermal, mechanical and biodegradation studies of biofiller based poly-3hydroxybutyrate biocomposites, Int. J. Biol. Macromol. (2019), doi:10.1016/j. ijbiomac.2019.11.112.

[77] E. Rudnik, D. Briassoulis, Comparative biodegradation in soil behaviour of two biodegradable polymers based on renewable resources, J. Polym. Environ. 19 (1) (2011) 18-39, doi:10.1007/s10924-010-0243-7.

[78] R. Rehman, "Selection of potent bacterial strain for over-production of PHB by using low cost carbon source for eco-friendly bioplastics," Nov. 2015.

[79] N. Teramoto, K. Urata, K. Ozawa, M. Shibata, Biodegradation of aliphatic polyester composites reinforced by abaca fiber, Polym. Degrad. Stab. 86 (3) (2004) 401-409 https://doi.org/10.1016/j.polymdegradstab.2004.04.026.

[80] A. Manna, A.K. Paul, Degradation of microbial polyester poly(3hydroxybutyrate) in environmental samples and in culture, Biodegradation 11 (5) (2000) 323-329, doi:10.1023/A:1011162624704.

[81] X. Wen, X. Lu, Microbial degradation of poly(3-hydroxybutyrate-co-4hydroxybutyrate) in soil, J. Polym. Environ. 20 (2) (2012) 381-387, doi:10. 1007/s10924-011-0387-0.

[82] S. Wang, C. Song, W. Mizuno, M. Sano, M. Maki, C. Yang, B. Zhang, S. Takeuchi, Estimation on biodegradability of poly (3-hydroxybutyrate-co-3hydroxyvalerate) (PHB/V) and numbers of aerobic PHB/V degrading microorganisms in different natural environments, J. Polym. Environ. 13 (1) (2005) 39-45, doi:10.1007/s10924-004-1214-7.

[83] S. Muniyasamy, O. Ofosu, M. John, R. Anandjiwala, Mineralization of poly (lactic acid)(PLA), poly (3-hydroxybutyrate-co-valerate)(PHBV) and PLA/PHBV blend in compost and soil environments, J. Renew. Mater. 4 (Jan.) (2016) 133-145

[84] B. Rani-Borges, A. Uemura Faria, A. de Campos, S.P. Costa Gonçalves, S.M. Martins-Franchetti, Biodegradation of additive PHBV/PP-co-PE films buried in soil, Polímeros Ciência e Tecnol 26 (Jan. 2) (2016) 161-167.

[85] L. Montagna, Evaluating the biodegradation of poly(3-hydroxybutyrate-co-3hydroxyvalerate)/carbon nanotube nanocomposites in soil. 2016.

[86] C.-S. Wu, Assessing biodegradability and mechanical, thermal, and morphological properties of an acrylic acid-modified poly(3-hydroxybutyric acid)/wood flours biocomposite, J. Appl. Polym. Sci. 102 (Nov. 4) (2006) 35653574, doi:10.1002/app.24817.

[87] H. Barragán, A. Pelacho, L. Martín-Closas, Degradation of agricultural biodegradable plastics in the soil under laboratory conditions, Soil Res. 54 (Feb.) (2016), doi:10.1071/SR15034.

[88] P. Rizzarelli, M. Cirica, G. Pastorelli, C. Puglisi, G. Valenti, Aliphatic poly(ester amide)s from sebacic acid and aminoalcohols of different chain length: synthesis, characterization and soil burial degradation, Polym. Degrad. Stab. 121 (2015) 90-99 https://doi.org/10.1016/j.polymdegradstab.2015.08.010.

[89] S. Casarin, S. Malmonge, F. Rosário, Biodegradable PHB/copolyester blends - biodegradation in soil, Polímeros 23 (Dec.) (2012) 115-122, doi:10.1590/ S0104-14282013005000003.

[90] S.O. Kulkarni, P.P. Kanekar, J.P. Jog, P.A. Patil, S.S. Nilegaonkar, S.S. Sarnaik, P.R. Kshirsagar, Characterisation of copolymer, poly (hydroxybutyrateco-hydroxyvalerate) (PHB-co-PHV) produced by Halomonas campisalis (MCM B-1027), its biodegradability and potential application, Bioresour. Technol. 102 (11) (2011) 6625-6628 https://doi.org/10.1016/j.biortech.2011.03.054

[91] L. Jeszeova, A. Puskarova, M. Buckova, L. Krakova, T. Grivalsky, M. Danko, K. Mosnackova, S. Chmela, D. Pangallo, Microbial communities responsible for the degradation of poly(lactic acid)/poly(3-hydroxybutyrate) blend mulches in soil burial respirometric tests, World J. Microbiol. Biotechnol. 34 (Jun. 7) (2018) 101, doi:10.1007/s11274-018-2483-y.

[92] V.E. Carofiglio, P. Stufano, N. Cancelli, V.M. De Benedictis, D. Centrone, E. De. Benedetto, A. Cataldo, A. Sannino, C. Demitri, Novel PHB/Olive mill wastewater residue composite based film: thermal, mechanical and degradation properties, J. Environ. Chem. Eng. 5 (6) (2017) 6001-6007 https://doi.org/10.1016/ j.jece.2017.11.013

[93] S. Dey, P. Tribedi, Microbial functional diversity plays an important role in the degradation of polyhydroxybutyrate (PHB) in soil, 3 Biotech. 8 (2018) 171, doi:10.1007/s13205-018-1201-7.

[94] S. Schröpfer, B.M. Karpinski, L. Bianchin, L. Robinson, V. Lima, V. Jahno, H. Barud, S. Ribeiro, Biodegradation evaluation of bacterial cellulose, vegetable cellulose and poly (3-hydroxybutyrate) in soil, Polímeros 25 (Mar.) (2015) 154-160, doi:10.1590/0104-1428.1712.

[95] S. Baidurah, P. Murugan, K.Y. Sen, Y. Furuyama, M. Nonome, K. Sudesh, Y. Ishida, Evaluation of soil burial biodegradation behavior of poly(3hydroxybutyrate-co-3-hydroxyhexanoate) on the basis of change in copolymer composition monitored by thermally assisted hydrolysis and methylation-gas chromatography, J. Anal. Appl. Pyrol. 137 (2019) 146-150 https://doi.org/10.1016/j.jaap.2018.11.020.
[96] C.-S. Wu, H.-T. Liao, Y.-X. Cai, Characterisation, biodegradability and application of palm fibre-reinforced polyhydroxyalkanoate composites, Polym. Degrad. Stab. 140 (2017) 55-63 https://doi.org/10.1016/j.polymdegradstab.2017. 04.016 .

[97] C.S. Wu, Preparation, characterization and biodegradability of crosslinked tea plant-fibre-reinforced polyhydroxyalkanoate composites, Polym. Degrad. Stab. (2013), doi:10.1016/j.polymdegradstab.2013.04.013.

[98] P. Mesquita, R. Araújo, D. Andrade, L. Carvalho, T. Alves, R. Barbosa, Evaluation of biodegradation of PHB/PP-G-MA/vermiculite bionanocomposites, Mater. Sci. Forum 869 (Aug.) (2016) 298-302 https://doi.org/10.4028/www. scientific.net/MSF.869.298.

[99] S.P.C. Gonçalves, M. Strauss, D.S.T. Martinez, The positive fate of biochar addition to soil in the degradation of PHBV-silver nanoparticle composites, Environ. Sci. Technol. 52 (Dec. 23) (2018) 13845-13853, doi:10.1021/acs.est. 8 b01524.

[100] A.N. Boyandin, S.V. Prudnikova, V.A. Karpov, V.N. Ivonin, N.L. Đỗ, T.H. Nguyễn, T.M.H. Lê, N.L. Filichev, A.L. Levin, M.L. Filipenko, T.G. Volova, I.I. Gitelson, Microbial degradation of polyhydroxyalkanoates in tropical soils, Int. Biodeterior. Biodegrad. 83 (2013) 77-84 https://doi.org/10.1016/j.ibiod.2013.04.014.

[101] K. Zhalnina, R. Dias, P.D. de Quadros, A. Davis-Richardson, F.A.O. Camargo, I.M. Clark, S.P. McGrath, P.R. Hirsch, E.W. Triplett, Soil pH determines microbial diversity and composition in the park grass experiment, Microb. Ecol. (2014), doi:10.1007/s00248-014-0530-2.

[102] A. Schirmer, C. Matz, D. Jendrossek, Substrate specificities of poly(hydroxyalkanoate)-degrading bacteria and active site studies on the extracellular poly(3-hydroxyoctanoic acid) depolymerase of Pseudomonas fluorescens GK13, Can. J. Microbiol. 41 (Suppl 1) (1995) 170-179, doi:10.1139/m95-184.

[103] S.-P. Lim, S.-N. Gan, I.K.P. Tan, Degradation of medium-chain-length polyhydroxyalkanoates in tropical forest and mangrove soils, Appl. Biochem. Biotechnol. 126 (Jul. 1) (2005) 23-33, doi:10.1007/s12010-005-0003-7.

[104] L. Joyyi, M.Z.A. Thirmizir, M.S. Salim, L. Han, P. Han, P. Murugan, K.-I. Kasuya, F.H.J. Maurer, M.I.Z. Arifin, K. Sudesh, Composite properties and biodegradation of biologically recovered $\mathrm{P}(3 \mathrm{HB}-\mathrm{co}-3 \mathrm{HHx})$ reinforced with short kenaf fibers, Polym. Degrad. Stab. 137 (2017) 100-108 https://doi.org/10.1016/ j.polymdegradstab.2017.01.004.

[105] S.-P. Yew, H.-Y. Tang, K. Sudesh, Photocatalytic activity and biodegradation of polyhydroxybutyrate films containing titanium dioxide, Polym. Degrad. Stab. 91 (8) (2006) 1800-1807 https://doi.org/10.1016/j.polymdegradstab.2005.11. 011

[106] N. Altaee, G.A. El-Hiti, A. Fahdil, K. Sudesh, E. Yousif, Biodegradation of different formulations of polyhydroxybutyrate films in soil, Springerplus 5 (1) (2016) 762, doi:10.1186/s40064-016-2480-2.

[107] Y.-X. Weng, L. Wang, M. Zhang, X.-L. Wang, Y.-Z. Wang, Biodegradation behavior of $\mathrm{P}(3 \mathrm{HB}, 4 \mathrm{HB}) / \mathrm{PLA}$ blends in real soil environments, Polym. Test. 32 (1) (2013) 60-70 https://doi.org/10.1016/j.polymertesting.2012.09.014.

[108] K. Kuntanoo, S. Promkotra, Biodegradation of polyhydroxybutyrate-co- hydroxyvalerate (PHBV) blended with natural rubber in soil environment, World Acad. Sci. Eng. Technol. 7 (Jan.) (2013) 1799-1803.

[109] S. Madbouly, J Schrader, G Srinivasan, K Liu, K McCabe, D Grewell, W Graves, M. Kessler, Biodegradation behavior of bacterial-based polyhydroxyalkanoate (PHA) and DDGS composites, Green Chem. 16 (Mar.) (2014), doi:10.1039/ C3GC41503A.

[110] F. Masood, T. Yasin, A. Hameed, Comparative oxo-biodegradation study of poly-3-hydroxybutyrate-co-3-hydroxyvalerate/polypropylene blend in controlled environments, Int. Biodeterior. Biodegrad. 87 (2014) 1-8 https://doi. org/10.1016/j.ibiod.2013.09.023.

[111] Z. Zaidi, D. Mawad, A. Crosky, Soil biodegradation of unidirectional polyhydroxybutyrate-co-valerate (PHBV) biocomposites toughened with polybutylene-adipate-co-terephthalate (PBAT) and epoxidized natural rubber (ENR), Front. Mater. 6 (2019) 275.

[112] N.M. Barkoula, S.K. Garkhail, T. Peijs, Biodegradable composites based on flax/polyhydroxybutyrate and its copolymer with hydroxyvalerate, Ind. Crops Prod. 31 (1) (2010) 34-42 https://doi.org/10.1016/j.indcrop.2009.08.005.

[113] A.W. Ajmal, F. Masood, T. Yasin, Influence of sepiolite on thermal, mechanical and biodegradation properties of poly-3-hydroxybutyrate-co-3hydroxyvalerate nanocomposites, Appl. Clay Sci. 156 (2018) 11-19 https://doi. org/10.1016/j.clay.2018.01.010.

[114] P. Mousavioun, G.A. George, W.O.S. Doherty, Environmental degradation of lignin/poly(hydroxybutyrate) blends, Polym. Degrad. Stab. 97 (7) (2012) 1114 1122 https://doi.org/10.1016/j.polymdegradstab.2012.04.004.

[115] H.Y. Sintim, A.I. Bary, D.G. Hayes, L.C. Wadsworth, M.B. Anunciado, M.E. English, S. Bandopadhyay, S.M. Schaeffer, J.M. DeBruyn, C.A. Miles, J.P. Reganold, M. Flury, In situ degradation of biodegradable plastic mulch films in compost and agricultural soils, Sci. Total Environ. 727 (2020) 138668 https://doi.org/ 10.1016/j.scitotenv.2020.138668.

[116] K. Sudesh, H. Abe, Y. Doi, Synthesis, structure and properties of polyhydroxyalkanoates: biological polyesters, Prog. Polym. Sci. 25 (10) (2000) 1503-1555 https://doi.org/10.1016/S0079-6700(00)00035-6.

[117] M. Knoll, T.M. Hamm, F. Wagner, V. Martinez, J. Pleiss, The PHA depolymerase engineering database: a systematic analysis tool for the diverse family of polyhydroxyalkanoate (PHA) depolymerases, BMC Bioinform. 10 (Mar.) (2009) 89, doi:10.1186/1471-2105-10-89.

[118] D. Jendrossek, A. Schirmer, H.G. Schlegel, Biodegradation of polyhydroxyalkanoic acids, Appl. Microbiol. Biotechnol. 46 (5) (1996) 451-463, doi:10.1007/ s002530050844. 
[119] B.I. Sang, K. Hori, Y. Tanji, H. Unno, Fungal contribution to in situ biodegradation of poly(3-hydroxybutyrate-co-3-hydroxyvalerate) film in soil, Appl. Microbiol. Biotechnol. 58 (Feb. 2) (2002) 241-247, doi:10.1007/ s00253-001-0884-5.

[120] V. Sultanpuram, T. Mothe, S.K. Mahmood, Biodegradation of polyhydroxyalkanoates, Internet J. Microbiol. 4 (Jan.) (2008) 1-6.

[121] M. Matavulj, H.P. Molitoris, Fungal degradation of polyhydroxyalkanoates and a semiquantitative assay for screening their degradation by terrestrial fungi, FEMS Microbiol. Rev. 9 (Dec. 2-4) (1992) 323-331, doi:10.1111/j.1574-6968. 1992.tb05854.x.

[122] H. Nishida, Y. Tokiwa, Effects of higher-order structure of poly(3hydroxybutyrate) on its biodegradation. II. Effects of crystal structure on microbial degradation, J. Environ. Polym. Degrad. 1 (1) (1993) 65-80, doi:10. 1007/BF01457654.
[123] C. Song, S. Wang, S. Ono, B. Zhang, C. Shimasaki, M. Inoue, The biodegradation of poly(3-hydroxybutyrate-co-3-hydroxyvalerate) (PHB/V) and PHB/V-degrading microorganisms in soil, Polym. Adv. Technol. 14 (Mar.) (2003) 184-188

[124] D. Akmal, M.N. Azizan, M.I.A. Majid, Biodegradation of microbial polyesters $\mathrm{P}(3 \mathrm{HB})$ and $\mathrm{P}(3 \mathrm{HB}-\mathrm{co}-3 \mathrm{HV})$ under the tropical climate environment, Polym. Degrad. Stab. 80 (3) (2003) 513-518 https://doi.org/10.1016/S0141-3910(03) 00034-X.

[125] A.N. Boyandin, V.P. Rudnev, V.N Ivonin, S.V. Prudnikova, K.I. Korobikhina, M.L. Filipenko, T.G. Volova, A.J. Sinskey, Biodegradation of polyhydroxyalkanoate films in natural environments, Macromol. Symp. 320 (Oct. 1) (2012) 38-42, doi:10.1002/masy.201251004. 